\title{
Plataformas digitales en la educación a distancia en México, una alternativa de estudio en comunicación
}

\section{Digital platforms in distance education in Mexico, a communication study alternative}

\author{
Arturo Secundino Hernández Gómez \\ Universidad Autónoma de Tamaulipas. Tampico, México. \\ ahernandez@docentes.uat.edu.mx \\ Ennio Héctor Carro Pérez \\ Universidad Autónoma de Tamaulipas. Tampico, México. \\ ecarro@docentes.uat.edu.mx \\ Isaías Martínez Trejo \\ Universidad Autónoma de Tamaulipas. Tampico, México. \\ imartint@docentes.uat.edu.mx
}

\begin{abstract}
Resumen
El presente estudio tiene como objetivo conocer la cantidad y características asociadas a la autoría, temática y de diseño de los estudios publicados sobre las plataformas digitales en la educación a distancia en México como una alternativa de estudio en la comunicación. La metodología empleada fue un diseño de investigación no experimental, transversal descriptivo. La población se conformó de 497 artículos obtenidos de las bases de datos DOAJ, SciELO y Redalyc, de los cuales solo siete artículos cumplieron con los criterios de inclusión para la muestra y fueron artículos que tuvieron un apartado metodológico, fueran escritos en español, inglés o portugués. Los resultados indican que de los 497 artículos encontrados, el 12\% de la población de artículos fueron encontrados en DOAJ, el $46 \%$ en SciELO y el $42 \%$ en Radalycl, los cuales fueron escritos en promedio por 2.5 autores. Conclusiones: se encontraron 7 artículos de la temática investigada, 5 corresponden a estudios empíricos y 2 de tipo teórico; el 60\% se realizan en la Universidad de Guadalajara, 20\% en la UNAM y $20 \%$ en la UAEH.
\end{abstract}

Palabras clave: Educación, plataforma, en línea, abierto, virtual.

\begin{abstract}
This study aims to know the quantity and characteristics associated with authorship, thematic and design of published studies on digital platforms in distance education in Mexico as an alternative study in communication. The methodology used was a nonexperimental, descriptive transversal research design. The population consisted of 497 articles obtained from the DOAJ, SciELO and Redalyc databases, of which only seven articles met the inclusion criteria for the sample and were articles that had a methodological section, were written in Spanish, English or Portuguese. The results indicate that of the 497 articles found, $12 \%$ of the population of articles were found in DOAJ, $46 \%$ in SciELO and $42 \%$ in Radalycl, which were written on average by 2.5 authors. Conclusions: 7 articles of the investigated subject were found, 5 correspond to empirical studies and 2 of theoretical type; $60 \%$ are made in the University of Guadalajara, $20 \%$ in the UNAM and $20 \%$ in the UAEH.
\end{abstract}

Keywords: Education, platform, online, open, virtual. 


\section{Introducción}

Las plataformas virtuales, son también un medio de comunicación que se emplea en el proceso formativo de la enseñanza-aprendizaje, sobre todo si se da en el ámbito universitario por los distintos tipos de plataformas digitales con características similares que existen y que son utilizadas como apoyo educativo; poseen además también distintas características como la comunicación sincrónica que emplea el chat, la videoconferencia o la pizarra compartida; o la comunicación asincrónica en donde se aplica el correo electrónico, los foros de debate y el tablón de anuncios, solo por mencionar algunas de los diseños más comunes dentro de una plataforma digital y que ambos tipos de comunicación, son importantes para el proceso formativo en la enseñanza-aprendizaje. Otra característica en las plataformas virtuales, es la interacción comunicativa que se da entre los distintos miembros de una comunidad virtual cuando hacen uso de ésta, y que puede generar información como el tipo de interacción que se da entre el profesor y los estudiantes o si la información proporcionada es útil al objetivo del proceso formativo de los estudiantes. Por ello, es necesario visualizar cuál es el tratamiento que dan los investigadores de la comunicación humana a la producción científica de artículos sobre plataformas digitales de enseñanza universitaria en México como una alternativa de estudio en comunicación.

En este sentido, los grandes avances tecnológicos han proporcionado una gran cantidad de información en todos los ámbitos del conocimiento, incluyendo los datos que aportan las investigaciones científicas y cualquier tipo de aporte a la ciencia, por lo que resulta extremadamente complicado estar actualizado sobre algún tema de estudio, porque cada día se publica más información (Moreno, Muñoz, Cuéllar, Domancic y Villanueva, 2018; Rubio-Aparicio, Sánchez-Meca, Marín-Martínez y López-López, 2018), por ello, frente a esta situación, es importante contar con herramientas que permitan la condensación de manera rigurosa de la información creciente, como son los estudios de síntesis científica de la información entre los que destacan las revisiones sistemáticas que resumen y renuevan los datos acreditados disponibles. Sin embargo, como todas las publicaciones de carácter científico, deben calificarse minuciosamente para conocer el nivel de validez que éstas presentan (Letelier, Manríquez y Rada, 2005) y por ello, se presenta en primer lugar, diversas definiciones sobre lo que se considera que es una revisión sistemática de la literatura, de acuerdo con las concepciones de los diversos autores que han realizado estudios de este tipo.

Las revisiones sistemáticas son investigaciones científicas a partir del análisis de los artículos originales que se han publicado hasta el momento en un determinado campo del conocimiento (Ferreira, Urrútia y Alonso-Coello, 2011); o como algunos investigadores que la consideran un estudio donde se resume, estructura, explica y sistematiza la información más relevante de los artículos analizados (Vidal, Oramas y Borroto, 2015 y Aguilera, 2014); y para aquellos autores que estiman que este tipo de investigación proporciona una síntesis muy completa y, desde un punto de vista descriptivo y objetivo de una gran cantidad de estudios relevantes (Torres-Fonseca y López-Hernández, 2014; Mombaque, Regina y Alves, 2018; Salvador-Oliván, Marco-Cuenca y Arquero-Avilés, 2018).

Por otra parte, la mayoría de los autores coinciden en que la revisión sistemática se desarrolla a partir de una serie de etapas como la definición de la pregunta de interés o investigación, los criterios de inclusión y exclusión, así como la detección y selección de los artículos a analizar, cómo obtener la información de los artículos y cómo presentar los Plataformas digitales en la educación a distancia en México, una alternativa de estudio en comunicación. Arturo S. Hernández, Ennio H. Carro y Isaías Martínez.

Página 2 de 27 
resultados obtenidos (Letelier, Manrique y Rada, 2005; Aguilera, 2014); sin embargo, algunos los detallan en forma de características o como objetivos de investigación a seguir al momento de realizar la revisión sistemática (Torres-Fonseca y López-Hernández, 2014).

Sin embargo, quienes definen mejor lo que es una revisión sistemática son CardonaArias, Higuita-Gutiérrez y Ríos Osorio (2016), al plantear que es un proceso de identificación sistemático que evalúa diversos publicaciones de una misma temática con el objetivo de resumir a través de un método científico la literatura analizada que permite que otros investigadores reproduzcan el estudio, por su parte Beltrán (2005) especifica que, dentro de la revisión sistemática existen dos formas de realizarlas, la cuantitativa o tipo metanálisis y la cualitativa o también llamada overview; por lo tanto, clarifica que, la diferencia entre estos dos tipos de revisiones sistemáticas radica en el uso y aplicación de la estadística que permite un análisis cuantitativo de los resultados, en general con el fin de calcular tamaños de efecto, de igual manera refiere que el concepto de meta-análisis fue acuñado por Glass en el año de 1976, quien planteó que este tipo de estudios meta-analíticos son una serie de análisis estadísticos a partir de los datos o análisis de un grupo o conjunto de estudios empíricos que comparten una temática particular de los resultados de estudios de cada artículo que conjuntan a su vez una serie de hallazgos en sus generalidades, por lo que es común que se utilice indistintamente el término meta-análisis y revisión sistemática cuando implican productos diferentes, el primero intenta obtener tamaño de efectos globales o promedio de las variables independientes observadas en los estudios y, el segundo, busca describir el conjunto de estudios a través de indicadores que van desde los de autoría y temática de trabajo hasta los metodológicos sin plantear análisis estadísticos causales de los resultados, por lo tanto, no son estudios iguales en su aplicación.

\section{Marco referencial}

Los recientes estudios sobre las plataformas digitales como apoyo a la educación universitaria en México, enfocan sus investigaciones en los modelos de enseñanza pedagógicos o sobre el proceso formativo a distancia como plantean Ramírez y Barajas (2017) o De Pablos, Colás, López y García-Lázaro (2019); los primeros han estudiado el impacto del uso de las plataformas educativas en la práctica pedagógica en el nivel superior educativo y, centran su atención en aspectos relativos a los modelos pedagógicos como el transmisivo, el de transición y el integrador como alternativas del proceso enseñanzaaprendizaje en dos instituciones educativas del estado de San Luis Potosí; y los segundos, estudiaron el uso de las plataformas digitales en la enseñanza universitaria desde la perspectiva de la investigación educativa como un proceso formativo basado en la presencialidad por otro más flexible o mixto basado en la actividad en línea (a distancia) y centrado en el desarrollo de materiales digitales para cursos on line en plataformas tipo Blackboard, Moodle, Formare o WebCT, en los sistemas universitarios como proceso de enseñanza-aprendizaje; en la misma línea de investigación, pero centrados en estudios sobre la valoración que hacen los estudiantes de nivel medio superior sobre el uso de la plataforma Moodle, como recurso de apoyo a las actividades de aprendizaje en la asignatura de informática en un Centro de Educación Media Superior de México, con la finalidad de generar información sobre el diseño de cursos con apoyos de sistemas de gestión de aprendizaje (Vidrio, Gómez y Zambrano, 2015). Por otra parte, existe un estudio dirigido a investigar cuál es el papel de la tecnología en el logro de los fines de la educación, mediante

Plataformas digitales en la educación a distancia en México, una alternativa de estudio en comunicación. Arturo S. Hernández, Ennio H. Carro y Isaías Martínez.

Página 3 de 27 
el uso sistemático de la tecnología educativa empleando medios tradicionales como libros, cuadernos apuntes o, herramientas que ofrecen las tecnologías de la información y comunicación (TIC) a la cual se le considera en el estudio, como el equivalente de la tecnología educativa; cabe decir que es el único artículo que menciona a la comunicación, pero en un sentido tecnológico.

Por esta razón, los estudios de revisiones sistemáticas que se presentan a continuación, reportan características orientadas al campo de la comunicación y visualizan el tratamiento de la sistematización de la información de estos estudios, la manera de analizar y caracterizar los hallazgos encontrados, además de explorar diversos tipos de contenidos relacionados o no con el proceso formativo de la enseñanza-aprendizaje o de la comunicación humana. En este sentido, los estudios desarrollados por Castillo-Esparcia, Rubio y AlmansaMartínez (2012) aportan información relativa a 10 publicaciones indexadas en el Sciences Citation Index (SCI) la cual es una base de datos, en donde se encuentran contribuciones de diferentes tipos como artículos, ensayos, editoriales, cartas, discusiones y revisiones, entre otros documentos. Con esas consideraciones se tuvo en cuenta los criterios contemplados en el SCI para que una revista pueda ser incluida. Algunas de las características más estandarizadas son la periodicidad de la revista para evitar apariciones esporádicas, el cumplimiento de las normas internacionales con ISSN que conllevan un título en el artículo que responda a la temática de la revista, datos de todos los autores o colaboradores, resumen y las palabras claves en idioma original e inglés, un proceso de revisión anónima por pares y que las revistas tengan relevancia en su campo científico. Por otra parte, Repiso, Torres y Delgado (2011) hacen una revisión de las tesis doctorales en televisión como objeto de análisis, comentando que éstas constituyen uno de los mejores objetos de estudio que transparentan las tendencias y perspectivas de investigación de la comunicación con la producción de tesis doctorales en las universidades españolas, sobresaliendo la Universidad Complutense de Madrid, la cual tiene el mayor número de tesis doctorales producidas de televisión, frente a otras universidades de ese país; la que genera el mayor grupo de académicos en tribunales como directores y evaluadores de tesis, seguida de la Universidad Autónoma de Barcelona, la Universidad de Navarra y la Universidad de la Laguna que generan sus propios grupos y, en las cuales, se localiza a los profesores que estructuran el sistema de investigación español en televisión, lo cual da una perspectiva específica sobre dónde se genera en España el conocimiento sobre la televisión y cuáles son las Universidades donde se genera y qué investigadores la generan.

Por lo tanto, se establecieron las siguientes preguntas de investigación:

¿Cuál es la producción de artículos empíricos y teóricos sobre la enseñanza universitaria con el apoyo de plataformas digitales en México?

¿Cuál es el promedio de autores, adscripción universitaria, nacionalidad e idiomas publicados sobre la enseñanza universitaria con el apoyo de plataformas digitales en México?

¿Cuáles son las características metodológicas de los artículos empíricos sobre la enseñanza universitaria con el apoyo de plataformas digitales en México?

Con base en lo anterior, algunos indicadores que se midieron en el presente estudio fueron los Indicadores de producción, es decir, se observó el número de revistas publicadas, la cantidad de autores por artículo, adscripción institucional, nacionalidad, los Indicadores

Plataformas digitales en la educación a distancia en México, una alternativa de estudio en comunicación. Arturo S. Hernández, Ennio H. Carro y Isaías Martínez.

Página 4 de 27 
de circulación o presencia de trabajos científicos en bases de datos bibliográficas; así como los Indicadores de la lengua que se utilizan en las revistas seleccionadas como el uso del idioma español, inglés, portugués u otros idiomas y los Indicadores metodológicos de los artículos empíricos, como el diseño de investigación, tipo de investigación y muestra.

Cuál es la producción de artículos empíricos y teóricos sobre la enseñanza universitaria con el apoyo de plataformas digitales en México

Asimismo, se planteó como objetivo general:

Conocer la cantidad de artículos empíricos y artículos teóricos, el promedio de autores, la adscripción institucional, la nacionalidad, el idioma y las características metodológicas de la producción de artículos publicados sobre la enseñanza universitaria con el apoyo de plataformas digitales en México.

En cuanto a los objetivos específicos:

Identificar y seleccionar la producción de artículos publicados sobre la enseñanza universitaria con el apoyo de plataformas digitales en México.

Clasificar los artículos empíricos y teóricos, la cantidad autores, la adscripción institucional de procedencia, la nacionalidad de procedencia y el idioma en que se han publicado sobre la enseñanza universitaria con el apoyo de plataformas digitales en México.

Comparar los artículos empíricos de acuerdo a sus características metodológicas que han sido publicados sobre la enseñanza universitaria con el apoyo de plataformas digitales en México.

En este sentido, se generó el siguiente planteamiento de hipótesis:

H1: La producción de artículos teóricos en la enseñanza universitaria con el apoyo de plataformas digitales en México, es moderada en comparación con la producción de artículos empíricos.

Ho: La producción de artículos teóricos en la enseñanza universitaria con el apoyo de plataformas digitales en México, no es moderada en comparación con la producción de artículos empíricos.

El incremento de la información en la educación tradicional como refiere Torres (2010) con el resurgimiento paulatino del humanismo en los campos educativos o de la expansión de la educación tecnológica a partir de los años setenta (Area, 1991), hace necesaria la realización de una revisión sobre la producción científica de la enseñanza universitaria con apoyo de plataformas digitales en México, la cual puede contribuir a ofrecer una radiografía sobre las prácticas de divulgación y publicación científica con el fin de conocer las universidades mexicanas que realizan trabajos de investigación, lo que podría permitir la elaboración de un diagnóstico sobre diversos aspectos: qué tipo de plataformas virtuales tienen las instituciones educativas; si se realizan estudios a partir de alguna de ellas y, si los hay, cuáles son los contenidos que más se abordan para su estudio, quiénes son los sujetos en los que se realiza el estudio, donde se centra la investigación: en la enseñanza o en el aprendizaje, o si el objeto de estudio se aborda en los contenidos, en la plataforma digital o, en los sujetos.

Plataformas digitales en la educación a distancia en México, una alternativa de estudio en comunicación. Arturo S. Hernández, Ennio H. Carro y Isaías Martínez.

Página 5 de 27 
También es importante la realización de la revisión para reconocer la cantidad de autores que realizan algún estudio sobre la enseñanza universitaria con el apoyo de plataformas digitales en México, lo que puede permitir elaborar una red de colaboración entre investigadores e instituciones de educación superior, tanto universitarias como tecnológicas, para ampliar el campo de investigación a nivel regional o institucional, así como crear una red de perfiles profesionales abocados a la investigación de las plataformas digitales de carácter multidisciplinar.

En el mismo sentido, conocer la publicaciones de artículos por país, también es importante, porque proporciona información específica sobre la producción de artículos en México y otros países, lo que da una visión integral documentada de cuáles son las temáticas que más se abordan en los estudios de la enseñanza universitaria con apoyo de plataformas digitales, pudiendo ofrecer nuevas líneas de acción para la investigación o, reforzar aquéllas líneas que están aún en estado incipiente de desarrollo; incluso, la obtención de información referente a la metodología aplicada a los trabajos de investigación en relación a la enseñanza universitaria con el apoyo de plataformas digitales, puede permitir elaborar un perfil de la investigación hecha hasta el momento, con lo que se estaría en posibilidad de señalar omisiones, malas y buenas prácticas metodológicas, costumbres investigativas y entonces indicar áreas de oportunidad o de trabajo futuro.

La relevancia del estudio será proporcionar una descripción sobre la producción científica en la enseñanza universitaria con apoyo de plataformas digitales en México, en un contexto donde dicha información existe, pero de manera dispersa, facilitando para ello datos e informes específicos para la comunidad científica, entre ellos, la del área de la comunicación y la educación.

Además de lo anterior, puede ser la vinculación de una red de trabajos entre diversos investigadores en el campo de la ciencia de la comunicación para la construcción de líneas de trabajo sobre el proceso comunicativo en la enseñanza-aprendizaje en plataformas digitales en instituciones de educación superior de México.

\section{Metodología}

La presente investigación es de corte descriptiva, debido a que se pretende explorar la producción y publicación de artículos en bases digitales científicas y describir la información encontrada acerca de las plataformas digitales en la educación a distancia en México.

Se plantea un diseño de investigación no experimental, de corte transversal. Lo anterior, considerando la temporalidad del estudio, en el que se recolecta en un solo momento los datos contenidos en los artículos a analizar, los cuales son el objeto del presente estudio (Díaz, 2007).

Por otra parte, al tratarse de una actividad para identificar, recolectar y analizar la información o literatura de acuerdo a lineamientos y métodos sistematizados, se considera que el estudio se realizará como una revisión sistemática con una metodología propia, de acuerdo a la propuesta por Gough, Oliver y Thomas (2012); en otras palabras, el estudio se

Plataformas digitales en la educación a distancia en México, una alternativa de estudio en comunicación. Arturo S. Hernández, Ennio H. Carro y Isaías Martínez.

Página 6 de 27 
acerca más a un mapeo sistemático (García-Peñalvo, 2017) totalmente descriptivo y de la que se refieren datos teóricos y metodológicos, así como temas de interés de un campo particular.

El universo de artículos encontrados fue de 497 y, se llegó a ellos, a través de diversas palabras clave relacionadas con la temática a investigar (plataforma, enseñanza, universidad, aprendizaje, educación) y, una vez seleccionadas, fueron filtradas en tres bases de datos digitales: Directory Open Acces Journal (DOAJ), Scientific Electronic Library Online (SciELO) y la Red de Revistas Científicas de América Latina, el Caribe, España y Portugal (Redalyc); dando como resultado esa cantidad de artículos publicados en una o más revistas entre los años 2000 al 2017. De esos 497 artículos, 61 de ellos se encontraron en DOAJ, 227 artículos se encontraron en SciELO y, 209 fueron encontrados en Redalyc.

De esos 497 artículos, se extrajo un conjunto de 37 artículos; los criterios para extraer ese conjunto de artículos fue que, el título de los artículos estuviera estrechamente relacionado con la enseñanza universitaria y las plataformas digitales en México, que los artículos fueran de corte empírico y, que fueran publicados en español, inglés y portugués.

Para la presente investigación se detectaron 37 artículos sobre las plataformas digitales en la educación a distancia en México, considerando el año de publicación de los artículos a partir del 2000 al año 2017. El tipo de muestreo realizado fue no probabilístico de tipo intencionado, realizándose una búsqueda exhaustiva en función de lo descrito en el procedimiento.

El procedimiento de análisis se llevó a cabo por fases, en la primera se determinaron las bases de datos digitales en las que se realizaría la búsqueda de los artículos primarios contemplándose las siguientes: el Directory of Open Access Journal (DOAJ), Scientific Electronic Library Online (SciELO) y Red de Revistas de América Latina, el Caribe, España y Portugal (Redalyc), seleccionadas a partir de su relevancia en la divulgación del conocimiento científico iberoamericano, incluidas en el portal del Consorcio Nacional de Recursos de Información Científica y Tecnológica (CONRICyT) del Consejo Nacional de Ciencia y Tecnología (CONACYT, 2019) de México, el cual las considera en la evaluación de revistas científicas, así mismo la Matriz de Información para el Análisis de Revistas (MIAR, 2019) de la Universidad de Barcelona, España, las toma en cuenta para la valoración de publicaciones científicas.

Los criterios empleados para la búsqueda de los artículos primarios relacionados con la temática a investigar son los que se enlistan: a) búsqueda por criterio-tema: Plataforma digital, plataformas digitales, plataforma virtual, plataformas virtuales, plataforma tecnológica y plataformas tecnológicas; b) búsqueda por criterio-palabras clave: plataforma, enseñanza; plataforma, universidad; plataforma, aprendizaje; plataforma, educación; plataforma, enseñanza, universidad y, plataforma, aprendizaje, universidad.

En la segunda fase se realizó la búsqueda de artículos vinculados a los criterios por temas y palabras clave filtradas en las tres bases de datos DOAJ, SciELO y Redalyc. En la tercera fase se elaboró una ficha de registro para recolectar la información referente a los

Plataformas digitales en la educación a distancia en México, una alternativa de estudio en comunicación. Arturo S. Hernández, Ennio H. Carro y Isaías Martínez.

Página 7 de 27 
artículos a analizar para después realizar la muestra a través de la agrupación de los artículos que tratasen exclusivamente sobre las plataformas digitales en la educación a distancia en México, a través del análisis de congruencia entre codificadores.

Durante la capacitación de los codificadores para la revisión de los artículos, se hizo lo siguiente: para agrupar y elaborar la categoría de artículos, se realizó un análisis denominado congruencia de codificadores en la cual se capacitaron a dos codificadores (codificador A y codificador B), considerando lo siguiente: seleccionar aquellos artículos en función de la temática sobre la enseñanza universitaria con apoyo de plataformas digitales en México, que el artículo tuviera un apartado metodológico o con características empíricas y estar publicado en español, inglés o portugués, para posteriormente, registrar el título del artículo en un formato de tabla donde se anotaban el título del artículo, la base de datos donde fue encontrado el artículo y una casilla para considerar "si" fue seleccionado el artículo por el codificador A, o "no" fue seleccionado el artículo por el mismo codificador A, lo mismo para el codificador B. Después se aplicó el coeficiente de Kappa como procedimiento para determinar la concordancia entre los dos codificadores (Siegel y Castellan, 1995).

En el proceso de probado de la confiabilidad de los codificadores a través del coeficiente Kappa, se realizó lo siguiente con la fórmula del índice de Kappa que es la que se observa a continuación:

$$
\mathrm{K}=\frac{\mathrm{P}(\mathrm{A})-\mathrm{P}(\mathrm{E})}{1-\mathrm{P}(\mathrm{E})}
$$

Donde:

$\mathrm{P}(\mathrm{A})=$ La proporción de concordancia observada (para este caso, la proporción de veces que los codificadores A y B concordaron en sus elecciones).

$\mathrm{P}(\mathrm{E})=$ La proporción de concordancia esperada por azar (para este caso, la proporción de veces esperada de que los codificadores A y B concuerden en sus elecciones por azar).

En donde los valores van de -1 a 1 y en la cual 0 es la concordancia esperada por el azar, -1 la mayor discordancia y 1 es mayor grado de acuerdo, como se describe en la propuesta de Landis y Koch (como se citó en Cerda y Villarroel, 2008), sobre cómo se interpreta cualitativamente el coeficiente de Kappa. Los resultados del coeficiente de kappa, se calcularon con el software SPSS-Statistics Visor (ver tabla 1), en donde los codificadores A y B, de acuerdo al gradiente -1 a 1, el valor de Kappa es de 0.233, el cual se considera por debajo de lo medio aceptable (fair), (García, 2015), lo que significa estadísticamente como un mínimo aceptable la concordancia entre los codificadores A y B, además de tener un valor aproximado de significancia de .090 , el cual está por arriba del .05

Tabla 1

Resultado del coeficiente Kappa para los codificadores $A$ y $B$

\begin{tabular}{lllc}
\hline & & Valor & Sig. aproximada \\
\hline Medida de acuerdo & Kappa & .233 & .099 \\
$\mathrm{~N}$ de casos válidos & & 180 & \\
\hline
\end{tabular}

Fuente: Autoría propia

Plataformas digitales en la educación a distancia en México, una alternativa de estudio en comunicación. Arturo S. Hernández, Ennio H. Carro y Isaías Martínez.

Página 8 de 27 


\section{Resultados}

El total de artículos encontrados por cada una de las bases de datos analizadas se observa en la tabla 2, así como el porcentaje representativo de cada una de éstas. SciELO fue la base de datos en la que se encontró un mayor número de artículos tal vez porque en ésta base digital se encuentran diversas revistas sobre educación y educación digital o virtual. Después, en menor medida pero igualando el cuarenta por ciento de artículos encontrados fue la base de datos Redalyc, en la que se esperaba encontrar el mayor número de artículos publicados; es importante señalar que de DOAJ, también se obtuvo una cantidad mínima de artículos para la conformación de los datos.

Tabla 2

Total de artículos encontrados por base de datos

\begin{tabular}{lcc}
\hline Base de datos & No. & \multicolumn{1}{c}{$\%$} \\
\hline DOAJ & 61 & $12 \%$ \\
SciELO & 227 & $46 \%$ \\
Redalyc & 209 & $42 \%$ \\
\multicolumn{1}{c}{ Totales } & 497 & $100 \%$ \\
\hline
\end{tabular}

Fuente: Autoría propia

Antes de iniciar a describir los resultados por bases de datos, es importante aclarar que el número de artículos encontrados aplicando el criterio de búsqueda por tema en la base de datos DOAJ (30 artículos, tabla 3) y, el número de artículos encontrados aplicando el criterio de búsqueda por palabras clave (31 artículos, tabla 4), suman un total de 61 artículos (12\%), cifra que se puede observar en la tabla 2, con un total de 497 artículos; lo mismo aplica para las bases de datos SciELO y Redalyc.

Una vez aclarado lo anterior, en la tabla 3 se observa que en la base digital SciELO se encontraron más cantidad de artículos, no así en Redalyc y DOAJ, cabe resaltar que Redalyc es una base de datos en donde todas las revistas contenidas en ella, son del campo de la comunicación, sin embargo, la cantidad de artículos encontrados fue de solo 64 artículos.

Tabla 3

Desglose de artículos encontrados con el criterio de búsqueda por temas

\begin{tabular}{lrrr}
\hline \multicolumn{1}{c}{ Criterio de búsqueda por tema } & \multicolumn{3}{c}{ Núm. de artículos encontrados } \\
\hline & DOAJ & Scielo & Redalyc \\
Plataforma digital & 8 & 16 & 5 \\
Plataforma tecnológica & 0 & 13 & 10 \\
Plataforma virtual & 11 & 35 & 17 \\
Plataformas digitales & 2 & 13 & 5 \\
Plataformas tecnológicas & 0 & 15 & 10 \\
Plataformas virtuales & 9 & 22 & 17 \\
$\quad$ Totales & 30 & 114 & 64 \\
\hline
\end{tabular}

Fuente: Autoría propia

Plataformas digitales en la educación a distancia en México, una alternativa de estudio en comunicación. Arturo S. Hernández, Ennio H. Carro y Isaías Martínez.

Página 9 de 27 
Por el contrario, en el criterio de búsqueda por palabras clave filtradas en las bases de datos, Redalyc tiene la mayor cantidad de artículos encontrados (ver tabla 4), sobre todo en las palabras filtradas plataforma-aprendizaje y plataforma-educación, seguida de la base de datos SciELO, con las palabras filtradas plataforma-aprendizaje, lo cual es importante porque se destaca que en ambas bases de datos existen suficientes artículos tanto teóricos como empíricos que tratan de manera general aspectos sobre la enseñanza y su apoyo en plataformas digitales.

Tabla 4

Desglose de artículos encontrados con el criterio de búsqueda por palabras clave Criterio de búsqueda por palabra clave Núm. de artículos

\begin{tabular}{lrrr} 
& \multicolumn{3}{c}{ encontrados } \\
\hline & DOAJ & Scielo & Redalyc \\
(plataforma) (aprendizaje) & 16 & 58 & 64 \\
(plataforma) (aprendizaje) (universidad) & 0 & 0 & 0 \\
(plataforma) (educación) & 7 & 17 & 52 \\
(plataforma) (enseñanza) & 8 & 25 & 19 \\
(plataforma) (enseñanza) (universidad) & 0 & 0 & 0 \\
(plataforma) (universidad) & 0 & 13 & 10 \\
\multicolumn{1}{c}{ Totales } & 31 & 113 & 145 \\
\hline
\end{tabular}

Fuente: Autoría propia

Por otra parte, se obtuvo la media de autores (ver tabla 5), al sumar el número de los mismos por cada artículo publicado (fueron 1,254 autores) entre el total de artículos encontrados en las bases digitales (que fue de 497 artículos), dando una media de 2.52 autores por artículo publicado sobre las diversas temáticas que abordan a las plataformas digitales en la educación a distancia, todos ellos vinculados a la enseñanza, educación, universidades o virtuales. Cabe destacar que había artículos que tenían uno o dos autores, pero también artículos que tenían tres, cinco, seis y hasta siete y ocho co-autores.

Tabla 5

Media de autores

\begin{tabular}{cc}
\hline Total de autores & Media \\
\hline 1,254 & 2.52 \\
\hline
\end{tabular}

Fuente: Autoría propia

En la tabla 6 se pude observar las diez instituciones o universidades de la que proceden, colaboran o están adscritos los autores que publican sobre plataformas de enseñanza, plataformas virtuales de educación o plataformas de aprendizaje, destacando la Universidad de Granada y la Universidad Distrital Francisco José de Caldas. Es importante señalar que las instituciones que se mencionan en la tabla 6, solo son las diez primeras instituciones de un listado de 459 artículos que reportan el nombre de la institución o universidad pero, también es necesario precisar que las siguientes instituciones educativas no aparecen en la lista de la tabla 6 por razones de espacio, y son la Universidad Autónoma del Estado de Hidalgo (UAEH) porque se encuentra en el lugar número 14 de dicho listado, al

Plataformas digitales en la educación a distancia en México, una alternativa de estudio en comunicación. Arturo S. Hernández, Ennio H. Carro y Isaías Martínez.

Página 10 de 27 
RED. Revista de Educación a Distancia. Núm. 59, Artíc. 07, 30-10-2019

DOI: http://dx.doi.org/10.6018/red/60/07

igual que la Universidad Nacional Autónoma de México (UNAM), la cual ocupa el número 24 de la lista y, la Universidad de Guadalajara, que está en el lugar 44 del listado.

Tabla 6

Instituciones o Universidades que más publican sobre la temática Institución o Universidad No. de \% artículos

\begin{tabular}{lrr} 
Universidad de Granada & 11 & 2 \\
Universidad Distrital Francisco José de Caldas & 9 & 2 \\
Universidad de la República & 7 & 2 \\
Universidad Nacional de Colombia & 7 & 2 \\
Instituto Tecnológico de Estudios Superiores de Occidente & 7 & 2 \\
Instituto Tecnológico de Morelia & 6 & 1 \\
Universidad de Sevilla & 6 & 1 \\
Universidad de Jaen y I. E. S. & 6 & 1 \\
Sociedad Colombiana de Anestesiología SCARE & 6 & 1 \\
Purdue University & 5 & 1 \\
\hline
\end{tabular}

Nota: Es importante señalar que, de los 497 artículos encontrados, solo 459 de ellos reportaron de qué institución procedían los autores y ese número se tomó para obtener el porcentaje correspondiente.

Fuente: Autoría propia

En la tabla 7, se pude observar el título de las revistas que más artículos publican acerca de la enseñanza-aprendizaje, plataformas virtuales, plataformas de enseñanza o plataformas de aprendizaje, destacando la revista Apertura, la cual publica diversas temáticas sobre la educación digital y tecnológica, la cual se encuentra en la base de datos SciELO, seguida de la revista Formación Universitaria y Pixel-Bit, que también tienen un perfil más orientado al campo de la educación digital y, le sigue la Revista RED de Educación a Distancia, que también está dentro del campo de la educación digital. Es importante señalar que las revistas que se mencionan en esta tabla, son las diez primeras de una lista más larga obtenida a partir de 485 artículos que reportan el nombre de la publicación y, por lo tanto, hay muchos títulos que no se mencionan, como la revista Apuntes que es la número 15 de la lista, Comunicar (17 de la lista), ReCIBE (18 de la lista), REXE (20 de la lista) o, Acta Paulista de Enfermería (51 de la lista), inInformación Research (101 de la lista) o Educ Educ (134 de la lista). Varias de éstas revistas se pueden observar en la tabla 10.

Tabla 7

Revistas con más artículos publicados sobre la temática

\begin{tabular}{lrr}
\hline \multicolumn{1}{c}{ Revistas } & $\begin{array}{r}\text { No. de } \\
\text { artículos }\end{array}$ & $\%$ \\
\hline Apertura & 34 & 7 \\
Formación Universitaria & 24 & 5 \\
Pixel-Bit. Revista de Medios y Educación & 16 & 3 \\
RED. Revista de Educación a Distancia & 13 & 3 \\
Actualidades Investigativas en Educación & 11 & 2 \\
Educación Médica & 9 & 2 \\
@tic: Revista d'Innovación Educativa & 8 & 2 \\
\hline
\end{tabular}

Plataformas digitales en la educación a distancia en México, una alternativa de estudio en comunicación. Arturo S. Hernández, Ennio H. Carro y Isaías Martínez.

Página 11 de 27 
RED. Revista de Educación a Distancia. Núm. 59, Artíc. 07, 30-10-2019

DOI: http://dx.doi.org/10.6018/red/60/07

\begin{tabular}{lrr}
\hline Sophia & 8 & 2 \\
Revista Electrónica Educare & 7 & 1 \\
Revista Brasileira de Enfermagem & 6 & 1 \\
\hline
\end{tabular}

Nota: Es importante señalar que, de los 497 artículos encontrados solo 485 reportan el nombre de la revista, por lo que este número se tomó para obtener el porcentaje total de las revistas con más artículos publicados.

Fuente: Autoría propia

En la tabla 8 se puede observar la cantidad de artículos encontrados que fueron publicados en distintos idiomas, así como su porcentaje; destaca el idioma español en donde existe una mayor publicación de temáticas relacionadas con la investigación, seguida del portugués y después el inglés.

Tabla 8

Idiomas en que más se publica sobre la temática investigada

\begin{tabular}{|c|c|c|c|}
\hline \multicolumn{2}{|c|}{ Idioma } & No. & $\%$ \\
\hline \multicolumn{2}{|l|}{ Español } & 310 & 62 \\
\hline \multirow{3}{*}{$\begin{array}{l}\text { Portugués } \\
\text { Inglés }\end{array}$} & & 106 & 21 \\
\hline & & 81 & 17 \\
\hline & Total & 497 & $100 \%$ \\
\hline
\end{tabular}

Fuente: Autoría propia

En la tabla 9 se observa el número y porcentaje correspondiente de artículos relacionados exclusivamente al tema de las plataformas digitales, enseñanza, instituciones educativas o universidades; también se observan los países que más publican sobre la enseñanza universitaria con apoyo de plataformas digitales, destacando Colombia, seguido de países como México, Chile y España. La selección se realizó en base a lo que se reporta en el artículo tomando en cuenta la nacionalidad del primer autor del estudio y que la investigación se haya efectuado en alguna escuela de nivel medio, medio superior o superior del país de origen, es decir, si el estudio se efectuó en una institución educativa de México, corresponde tomarlo como una publicación que se realiza en éste país.

Tabla 9

\begin{tabular}{|c|c|c|}
\hline País & No. & $\%$ \\
\hline Colombia & 9 & 24 \\
\hline México & 7 & 19 \\
\hline Chile & 5 & 14 \\
\hline España & 4 & 11 \\
\hline Argentina & 3 & 9 \\
\hline Brasil & 2 & 5 \\
\hline Perú & 2 & 5 \\
\hline Uruguay & 2 & 5 \\
\hline Costa Rica & 2 & 5 \\
\hline Cuba & 1 & 3 \\
\hline Total & 37 & $100 \%$ \\
\hline
\end{tabular}

Fuente: Autoría propia

Plataformas digitales en la educación a distancia en México, una alternativa de estudio en comunicación. Arturo S. Hernández, Ennio H. Carro y Isaías Martínez.

Página 12 de 27 
En la tabla 10 se observan los artículos teóricos y empíricos detectados, pero clasificados a partir de la identificación del país en donde se encuentra la institución o universidad que edita la revista, además del título de la revista; después, se observa el título del artículo y el autor o autores de dicho artículo, posteriormente el lugar de adscripción o trabajo del autor o autores; finalmente el tipo de artículo. De los artículos detectados en base a que se realizaron en alguna institución educativa de determinado país y, que el primer autor fuera de dicho país, están clasificados los siete artículos que hacen referencia a México, dos de ellos son teóricos, y se pueden observar en la tabla 10 en la columna de título del artículo, identificados con los números 2 y 14; los otros cinco artículos son de corte empírico y se pueden observar en la misma tabla 10 en la columna de título del artículo, identificados con los números16, 19, 23, 31 y 33. También es importante mencionar que, de acuerdo al estudio realizado en la institución educativa o universidad del país referenciado en la publicación, en algunos trabajos hay colaboraciones de otras instituciones extranjeras, así como de sus respectivos autores, México comparte un artículo empírico con la Universidad de Málaga, Colombia comparte otro artículo empírico con la Universidad de Barcelona y, España comparte un artículo empírico con la Universidad de Valparaíso, Chile.

Tabla 10

Artículos seleccionados bajo el análisis de congruencia codificadores

\begin{tabular}{|c|c|c|c|c|c|}
\hline País & $\begin{array}{l}\text { Institución que } \\
\text { edita la revista }\end{array}$ & $\begin{array}{l}\text { Título de la } \\
\text { revista }\end{array}$ & Título del artículo & $\begin{array}{l}\text { Autor(es) de artículo y } \\
\text { lugar de adscripción }\end{array}$ & $\begin{array}{l}\text { Tipo de } \\
\text { artículo }\end{array}$ \\
\hline España & $\begin{array}{l}\text { Fac. de Biblio- } \\
\text { conomía y Do- } \\
\text { cumentación } \\
\text { Univ. de } \\
\text { Granada }\end{array}$ & $\begin{array}{l}\text { iR Information } \\
\text { Research }\end{array}$ & $\begin{array}{l}\text { 1. Diseño y desarrollo de una } \\
\text { plataforma digital de apoyo a la } \\
\text { docencia y a la investigación en } \\
\text { archivos electrónicos: un portal } \\
\text { temático (Ruíz y Gómez, 2004). }\end{array}$ & $\begin{array}{l}\text { Facultad de } \\
\text { Biblioteconomía y } \\
\text { Documentación, } \\
\text { Universidad de } \\
\text { Granada }\end{array}$ & Teórico \\
\hline México & $\begin{array}{l}\text { Universidad } \\
\text { de } \\
\text { Guadalajara }\end{array}$ & $\begin{array}{l}\text { ReCIBE-Rev. } \\
\text { Electrónica, } \\
\text { Computación, } \\
\text { Informática, } \\
\text { Biomédica y } \\
\text { Electrónica }\end{array}$ & $\begin{array}{l}\text { 2. Diseño e implementación de una } \\
\text { plataforma digital para la gestión de } \\
\text { tutorías y su impacto en la } \\
\text { deserción de estudiantes de nivel } \\
\text { superior (Zulma, Vega, Nivardy y } \\
\text { Hernández, 2017). }\end{array}$ & $\begin{array}{l}\text { Dpto. de Sistemas y } \\
\text { Computación del } \\
\text { Instituto Tecnológico } \\
\text { de Morelia, } \\
\text { Michoacán; } \\
\text { Universidad Vasco de } \\
\text { Quiroga, Michoacán, } \\
\text { México }\end{array}$ & Teórico \\
\hline Brasil & $\begin{array}{l}\text { Asociación } \\
\text { Brasileña de } \\
\text { Enfermería }\end{array}$ & $\begin{array}{l}\text { Rev. Brasileira } \\
\text { de Enferma- } \\
\text { gem REBEn }\end{array}$ & $\begin{array}{l}\text { 4. Teoria da aprendizagem } \\
\text { Signifcativa: elaboração e avaliação } \\
\text { de aula virtual na plataforma } \\
\text { Moodle (Prado, Rodríguez y de } \\
\text { Almeida, 2011). }\end{array}$ & $\begin{array}{l}\text { Escuela de Enfermería } \\
\text { de la Universidad de } \\
\text { Sao Paulo }\end{array}$ & Teórico \\
\hline Colombia & $\begin{array}{l}\text { Universidad } \\
\text { Santo Tomás }\end{array}$ & Ingenio Magno & $\begin{array}{l}\text { 5. Plataforma virtual de péndulo } \\
\text { sobre carro deslizante para la } \\
\text { enseñanza del control automático } \\
\text { (Rodríguez y Pineda, 2011). }\end{array}$ & $\begin{array}{l}\text { Universidad } \\
\text { Pedagógica y } \\
\text { Tecnológica de } \\
\text { Colombia Sogamoso, } \\
\text { Colombia }\end{array}$ & Teórico \\
\hline
\end{tabular}

Plataformas digitales en la educación a distancia en México, una alternativa de estudio en comunicación. Arturo S. Hernández, Ennio H. Carro y Isaías Martínez.

Página 13 de 27 


\begin{tabular}{|c|c|c|c|c|c|}
\hline Perú & $\begin{array}{l}\text { Fondo } \\
\text { editorial de la } \\
\text { Univ. del } \\
\text { Pacífico }\end{array}$ & $\begin{array}{l}\text { Apuntes. Rev. } \\
\text { de ciencias } \\
\text { sociales }\end{array}$ & $\begin{array}{l}\text { 6. Optimización y evaluación de la } \\
\text { plataforma virtual continental para } \\
\text { mejorar la gestión de tutoría virtual } \\
\text { (Córdova, 2011). }\end{array}$ & $\begin{array}{l}\text { Docente del Instituto } \\
\text { Continental, Perú }\end{array}$ & Empírico \\
\hline España & $\begin{array}{l}\text { Universidad } \\
\text { de Valencia }\end{array}$ & $\begin{array}{l}\text { @tic rev. } \\
\text { d'innovació } \\
\text { educativa }\end{array}$ & $\begin{array}{l}\text { 7. La } e \text {-Evaluación del aprendizaje } \\
\text { a través de la plataforma Aula } \\
\text { Virtual de la Universitat de } \\
\text { València (Chiva, Ramos, Gómez y } \\
\text { Alonso, 2013). }\end{array}$ & $\begin{array}{l}\text { Profs. contratados del } \\
\text { Dpto. de Historia, } \\
\text { Didáctica y Métodos, } \\
\text { de la Universidad de } \\
\text { Valencia }\end{array}$ & Empírico \\
\hline Colombia & $\begin{array}{l}\text { Universidad } \\
\text { de Antioquía }\end{array}$ & $\begin{array}{l}\text { Rev. Interame- } \\
\text { ricana de } \\
\text { Bibliotecolo-gía }\end{array}$ & $\begin{array}{l}\text { 8. La Biblioteca de la Facultad de } \\
\text { Ingeniería en la plataforma } \\
\text { educativa de la UdelaR-Uruguay: } \\
\text { implementando un servicio de } \\
\text { formación de usuarios virtual } \\
\text { (Andrade y Velázquez, 2014). }\end{array}$ & $\begin{array}{l}\text { Docentes de la } \\
\text { Facultad de Ingeniería, } \\
\text { Universidad de la } \\
\text { República, Uruguay }\end{array}$ & Teórico \\
\hline Perú & $\begin{array}{l}\text { Fondo } \\
\text { Editorial de la } \\
\text { Univ. del } \\
\text { Pacífico }\end{array}$ & $\begin{array}{l}\text { Apuntes. Rev. } \\
\text { de ciencias } \\
\text { sociales }\end{array}$ & $\begin{array}{l}\text { 9. Impacto del uso de la plataforma } \\
\text { virtual Moodle en el proceso de } \\
\text { enseñanza aprendizaje en la } \\
\text { Universidad Continental (Santa, } \\
\text { Calderón y Rojas, 2011). }\end{array}$ & $\begin{array}{l}\text { Docentes de la } \\
\text { Universidad } \\
\text { Continental, Perú }\end{array}$ & Empírico \\
\hline España & $\begin{array}{l}\text { Fundación } \\
\text { Privada } \\
\text { Educación } \\
\text { Médica }\end{array}$ & Rev. Edu Med & $\begin{array}{l}\text { 10. Docencia universitaria } \\
\text { semipresencial. Experiencia en el } \\
\text { uso de la plataforma virtual SWAD } \\
\text { (López-Morales, Celma-Vicente, } \\
\text { Cano-Caballero, Quero-Rufián y } \\
\text { Rodríguez-López, 2011). }\end{array}$ & $\begin{array}{l}\text { Docentes de la Escuela } \\
\text { Universitaria de } \\
\text { Enfermería Virgen de } \\
\text { las Nieves. Universidad } \\
\text { de Granada }\end{array}$ & Teórico \\
\hline Colombia & $\begin{array}{l}\text { Fac. de Educa- } \\
\text { ción, Univ. de } \\
\text { la Sabana }\end{array}$ & Educ.Educ. & $\begin{array}{l}\text { 11. Aprendizaje autorregulado a } \\
\text { través de la plataforma virtual } \\
\text { Moodle (Valenzuela-Zambrano y } \\
\text { Pérez Villalobos, 2013). }\end{array}$ & $\begin{array}{l}\text { Docentes de la Univ. } \\
\text { de Concepción, Chile }\end{array}$ & Teoríco \\
\hline Chile & $\begin{array}{l}\text { Fac. de Educa- } \\
\text { ción de la } \\
\text { Univ. Católica } \\
\text { de la } \\
\text { Santísima } \\
\text { Concepción }\end{array}$ & $\begin{array}{l}\text { Rev. de } \\
\text { Estudios y } \\
\text { Experiencias en } \\
\text { Educación, } \\
\text { REXE }\end{array}$ & $\begin{array}{l}\text { 12. Análisis de la efectividad del } \\
\text { uso de la plataforma virtual webct } \\
\text { en el proceso de enseñanza y } \\
\text { aprendizaje en la Universidad del } \\
\text { Magdalena, Colombia (Pérez y } \\
\text { Saker, 2012). }\end{array}$ & $\begin{array}{l}\text { Universidad del } \\
\text { Magdalena Santa } \\
\text { Martha, Colombia }\end{array}$ & Empírico \\
\hline $\begin{array}{l}\text { Costa } \\
\text { Rica }\end{array}$ & $\begin{array}{l}\text { Universidad } \\
\text { de Costa Rica, } \\
\text { Escuela de } \\
\text { Bibliotecologí } \\
\text { a y Ciencias } \\
\text { de la } \\
\text { Información, }\end{array}$ & $\begin{array}{l}\text { e-Ciencias de la } \\
\text { información } \\
\text { Rev.electróni-ca } \\
\text { Universidad de } \\
\text { Costa Rica }\end{array}$ & $\begin{array}{l}\text { 13. ¿Cómo brindar servicios de } \\
\text { diseminación selectiva de la } \\
\text { información (D.S.I.) a través de las } \\
\text { plataformas de aprendizaje } \\
\text { Virtuales (P.A.V.)?: una propuesta } \\
\text { de convergencia de sistemas (Meza, } \\
\text { 2013). }\end{array}$ & $\begin{array}{l}\text { Carrera de } \\
\text { Archivística. } \\
\text { Universidad Estatal a } \\
\text { Distancia; Carrera de } \\
\text { Bibliotecología, } \\
\text { Universidad de Costa } \\
\text { Rica }\end{array}$ & Teórico \\
\hline Cuba & $\begin{array}{l}\text { Centro de } \\
\text { Estudios de } \\
\text { Didáctica de la } \\
\text { Universidad } \\
\text { de Las Tunas } \\
\text { (CEDUT), } \\
\text { Cuba. }\end{array}$ & $\begin{array}{l}\text { Didasc@lia: } \\
\text { Rev. Didáctica } \\
\text { y Educación. }\end{array}$ & $\begin{array}{l}\text { 14. La asesoría en la enseñanza de } \\
\text { las matemáticas en la educación a } \\
\text { distancia, utilizando la plataforma } \\
\text { Web CT (Carrillo y Mota, 2010). }\end{array}$ & $\begin{array}{l}\text { Docentes del Centro } \\
\text { Universitario del Norte. } \\
\text { Universidad de } \\
\text { Guadalajara. Colotlán } \\
\text { Jalisco, México }\end{array}$ & Teórico \\
\hline España & $\begin{array}{l}\text { Grupo } \\
\text { Comunicar, } \\
\text { España }\end{array}$ & $\begin{array}{l}\text { Revista } \\
\text { Comunicar }\end{array}$ & $\begin{array}{l}\text { 15. Competencias en los procesos } \\
\text { de enseñanza-aprendizaje virtual y } \\
\text { semipresencial (Imbernón, Silva y } \\
\text { Guzmán, 2011). }\end{array}$ & $\begin{array}{l}\text { Fac. de Pedagogía de la } \\
\text { Univ. de Barcelona y } \\
\text { Facultad de Medicina, } \\
\text { Univ. de Valparaíso, } \\
\text { Chile }\end{array}$ & Empírico \\
\hline
\end{tabular}

Plataformas digitales en la educación a distancia en México, una alternativa de estudio en comunicación. Arturo S. Hernández, Ennio H. Carro y Isaías Martínez.

Página 14 de 27 
RED. Revista de Educación a Distancia. Núm. 59, Artíc. 07, 30-10-2019

DOI: http://dx.doi.org/10.6018/red/60/07

\begin{tabular}{|c|c|c|c|c|c|}
\hline México & $\begin{array}{l}\text { Universidad } \\
\text { de } \\
\text { Guadalajara }\end{array}$ & Apertura & $\begin{array}{l}\text { 16. Perspectiva docente sobre el } \\
\text { ambiente de aprendizaje en el } \\
\text { sistema de educación a distancia de } \\
\text { la Facultad de Ingeniería en } \\
\text { Sistemas de Producción } \\
\text { Agropecuaria (Velázquez y } \\
\text { Martínez, 2011). }\end{array}$ & $\begin{array}{l}\text { Docentes de la } \\
\text { Universidad } \\
\text { Veracruzana }\end{array}$ & Empírico \\
\hline México & $\begin{array}{l}\text { Universidad } \\
\text { de } \\
\text { Guadalajara }\end{array}$ & Apertura & $\begin{array}{l}\text { 17. Estilos de aprendizaje basados } \\
\text { en el modelo de Kolb en la } \\
\text { educación virtual (Romero, Salinas } \\
\text { y Mortera, 2010). }\end{array}$ & $\begin{array}{l}\text { Corporación } \\
\text { Universitaria Minuto } \\
\text { de Dios, Colombia; } \\
\text { Universidad Virtual del } \\
\text { Tecnológico de } \\
\text { Monterrey. Monterrey, } \\
\text { N.L. México }\end{array}$ & Empírico \\
\hline México & $\begin{array}{l}\text { Universidad } \\
\text { Nacional } \\
\text { Autónoma de } \\
\text { México } \\
\text { (UNAM) }\end{array}$ & $\begin{array}{l}\text { Educación } \\
\text { Química }\end{array}$ & $\begin{array}{l}\text { 18. Uso de la plataforma } \\
\text { socrative.com para alumnos de } \\
\text { Química General (Frías, Arce y } \\
\text { Flores-Morales, 2015). }\end{array}$ & $\begin{array}{l}\text { Universidad Arturo } \\
\text { Prat, Iquique, Chile }\end{array}$ & Empírico \\
\hline México & $\begin{array}{l}\text { Universidad } \\
\text { de } \\
\text { Guadalajara }\end{array}$ & Apertura. & $\begin{array}{l}\text { 19. La comunicación y la } \\
\text { colaboración vistas a través de la } \\
\text { experiencia en un MOOC } \\
\text { (Vázquez, Bras, Bucio y Rodríguez, } \\
\text { 2017). }\end{array}$ & $\begin{array}{l}\text { Docentes de: } \\
\text { Coordinación de } \\
\text { Universidad Abierta y } \\
\text { Educación a Distancia, } \\
\text { UNAM; Universidad } \\
\text { Pedagógica Nacional, } \\
\text { México }\end{array}$ & Empírico \\
\hline Brasil & $\begin{array}{l}\text { Escuela } \\
\text { Paulista de } \\
\text { Enfermagem, } \\
\text { Univ. Fed. de } \\
\text { Sao Paulo, } \\
\text { Brasil }\end{array}$ & $\begin{array}{l}\text { Acta Paulista de } \\
\text { Enfermería }\end{array}$ & $\begin{array}{l}\text { 20. Moodle platform for the } \\
\text { construction of knowledge in } \\
\text { intensive care: an experimental } \\
\text { study (Lopes yRizzo, 2016). }\end{array}$ & $\begin{array}{l}\text { Escuela Paulista de } \\
\text { Enfermería, Sao Paulo, } \\
\text { Brasil }\end{array}$ & Empírico \\
\hline Colombia & $\begin{array}{l}\text { Universidad } \\
\text { de Medellín }\end{array}$ & Anagrama & $\begin{array}{l}\text { 21. Profesores frente a estudiantes: } \\
\text { las dos orillas de la educación } \\
\text { bimodal (Quinceno, Arango y } \\
\text { Vázquez, 2016). }\end{array}$ & $\begin{array}{l}\text { Universidad de } \\
\text { Medellín }\end{array}$ & Empírico \\
\hline Argentina & $\begin{array}{l}\text { Universidad } \\
\text { Nacional de } \\
\text { Rosario } \\
\text { Argentina }\end{array}$ & $\begin{array}{l}\text { La trama de la } \\
\text { Comunicación }\end{array}$ & $\begin{array}{l}\text { 22. Migración y TIC: Identidades } \\
\text { andinas en Facebook (Melella, } \\
\text { 2016). }\end{array}$ & $\begin{array}{l}\text { Universidad Nacional } \\
\text { de Buenos Aires, } \\
\text { Argentina }\end{array}$ & Empírico \\
\hline México & $\begin{array}{l}\text { Universidad } \\
\text { de } \\
\text { Guadalajara }\end{array}$ & Apertura. & $\begin{array}{l}\text { 23. Percepción del profesor sobre el } \\
\text { uso del b-learning para fortalecer } \\
\text { competencias laborales (Ocampo, } \\
\text { Gómez y Zambrano, 2015). }\end{array}$ & $\begin{array}{l}\text { (Universidad } \\
\text { Autónoma del Estado } \\
\text { de México; Instituto } \\
\text { Tecnológico de } \\
\text { Estudios Superiores de } \\
\text { Monterrey; Prepa en } \\
\text { línea-SEP, México }\end{array}$ & Empírico \\
\hline Brasil & $\begin{array}{l}\text { Fac. de Educa- } \\
\text { ción de la } \\
\text { Universidad } \\
\text { de Sao Paulo }\end{array}$ & $\begin{array}{l}\text { Educación e } \\
\text { investigación }\end{array}$ & $\begin{array}{l}\text { 24. Rendimiento académico e } \\
\text { interacción sociocognitiva de } \\
\text { estudiantes en un entorno virtua } \\
\text { (Borgobello y Roselli, 2016). }\end{array}$ & $\begin{array}{l}\text { Pontificia Universidad } \\
\text { católica de Argentina } \\
\text { Santa María de los BS. } \\
\text { AS; Universidad } \\
\text { Nacional del Rosario }\end{array}$ & Empírico \\
\hline
\end{tabular}

Plataformas digitales en la educación a distancia en México, una alternativa de estudio en comunicación. Arturo S. Hernández, Ennio H. Carro y Isaías Martínez. Página 15 de 27 
RED. Revista de Educación a Distancia. Núm. 59, Artíc. 07, 30-10-2019

DOI: http://dx.doi.org/10.6018/red/60/07

\begin{tabular}{|c|c|c|c|c|c|}
\hline Costa & Universidad & Revista & 25. Definición de aspectos & Universidad Estatal a & Empírico \\
\hline Rica & Nacional & $\begin{array}{l}\text { Electrónica } \\
\text { Educare }\end{array}$ & $\begin{array}{l}\text { procedimentales y buenas prácticas } \\
\text { para el servicio de asesoría del } \\
\text { programa aprendizaje en línea en la } \\
\text { Universidad Estatal a Distancia de } \\
\text { Costa Rica (Mora-Vicarioli, } \\
\text { Hooper-Simpson y Durán Gutiérrez } \\
(2017) \text {. }\end{array}$ & Distancia, Costa Rica & \\
\hline Colombia & $\begin{array}{l}\text { Universidad } \\
\text { La Gran } \\
\text { Colombia, } \\
\text { Seccional } \\
\text { Armenia }\end{array}$ & Sophia & $\begin{array}{l}\text { 26. Buenas prácticas en la } \\
\text { educación superior virtual a partir } \\
\text { de especificaciones de estándares e- } \\
\text { Learning (Carmona y Rodríguez, } \\
\text { 2017). }\end{array}$ & $\begin{array}{l}\text { Universidad de } \\
\text { Quindío; Universidad } \\
\text { Central de Colombia }\end{array}$ & Empírico \\
\hline Colombia & $\begin{array}{l}\text { Corporación } \\
\text { Universitaria } \\
\text { Lasallista, } \\
\text { Antioquía }\end{array}$ & $\begin{array}{l}\text { Revista } \\
\text { Lasallista de } \\
\text { investigación }\end{array}$ & $\begin{array}{l}\text { 27. Percepciones estudiantiles } \\
\text { acerca del uso de nuevas } \\
\text { tecnologías en instituciones de } \\
\text { Educación Superior en Medellín } \\
\text { (Chalela, Valencia, Bermúdez y } \\
\text { Ortega, 2016). }\end{array}$ & $\begin{array}{l}\text { Universidad Autónoma } \\
\text { de Barcelona, España; } \\
\text { Instituto Tecnológico } \\
\text { Metropolitano, } \\
\text { Colombia }\end{array}$ & Empírico \\
\hline México & $\begin{array}{l}\text { Universidad } \\
\text { Nacional } \\
\text { Autónoma de } \\
\text { México }\end{array}$ & $\begin{array}{l}\text { Educación } \\
\text { Química }\end{array}$ & $\begin{array}{l}\text { 28. Campus Virtual y Facebook en } \\
\text { el ámbito universitario. ¿Enemigos } \\
\text { o aliados en los procesos de } \\
\text { enseñanza y aprendizaje? (Mansilla, } \\
\text { Muscia y Ugliarolo, 2013) }\end{array}$ & $\begin{array}{l}\text { Fac. de Farmacia y } \\
\text { Bioquímica, Univ. de } \\
\text { Buenos Aires, } \\
\text { Argentina }\end{array}$ & Empírico \\
\hline Chile & $\begin{array}{l}\text { Sociedad } \\
\text { Chilena de } \\
\text { Anatomía }\end{array}$ & $\begin{array}{l}\text { International } \\
\text { Journal of } \\
\text { Morphology } \\
\text { (Revista } \\
\text { Internacional de } \\
\text { Morfología) }\end{array}$ & $\begin{array}{l}\text { 29. Anatomicis Network: una } \\
\text { Plataforma de software educativa } \\
\text { basada en la nube para mejorar la } \\
\text { enseñanza de la anatomía en la } \\
\text { educación médica (Inzunza, Neyem, } \\
\text { Sanz, Valdivia, Villarroel, Farfán, } \\
\text { Matte y López-Juri, 2017). }\end{array}$ & $\begin{array}{l}\text { Escuela de Medicina y } \\
\text { Escuela de Ingeniería } \\
\text { de la Pontificia } \\
\text { Universidad católica de } \\
\text { Chile; Fac. de } \\
\text { Medicina de la } \\
\text { Universidad Austral de } \\
\text { Chile; Fac. de Ciencias } \\
\text { de la Salud, } \\
\text { Universidad de } \\
\text { Antofogasta, Chile y } \\
\text { Fac. de C. Naturales de } \\
\text { la Univ. de Playa } \\
\text { Ancha, Valparaíso, } \\
\text { Chile }\end{array}$ & Empírico \\
\hline México & $\begin{array}{l}\text { Universidad } \\
\text { Nacional } \\
\text { Autónoma de } \\
\text { México }\end{array}$ & $\begin{array}{l}\text { Educación } \\
\text { Química }\end{array}$ & $\begin{array}{l}\text { 30. Wikis en Moodle: la mirada de } \\
\text { estudiantes y docentes (Núñez, } \\
\text { Míguez y Seoane, 2016). }\end{array}$ & $\begin{array}{l}\text { Fac. de Química y Fac. } \\
\text { de Ingeniería de la } \\
\text { Universidad de la } \\
\text { República, } \\
\text { Montevideo, Uruguay }\end{array}$ & Teórico \\
\hline Chile & $\begin{array}{l}\text { Centro de } \\
\text { Información } \\
\text { Tecnológica } \\
\text { (CIT), La } \\
\text { Serena }\end{array}$ & $\begin{array}{l}\text { Formación } \\
\text { Universitaria }\end{array}$ & $\begin{array}{l}\text { 31. Integración de las tecnologías } \\
\text { de la información y comunicación } \\
\text { en un modelo de enseñanza flexible. } \\
\text { El caso del Centro Universitario de } \\
\text { los Valles de Guadalajara, México } \\
\text { (Calderón-Mayorga, Ruiz-Palmero } \\
\text { y Sánchez-Rodríguez, 2016). }\end{array}$ & $\begin{array}{l}\text { Centro Universitario de } \\
\text { los Valles, Universidad } \\
\text { de Guadalajara, } \\
\text { México; Fac. de } \\
\text { Ciencias de la } \\
\text { Educación, } \\
\text { Universidad de } \\
\text { Málaga, España }\end{array}$ & Empírico \\
\hline Chile & $\begin{array}{l}\text { Centro de } \\
\text { Información } \\
\text { Tecnológica }\end{array}$ & $\begin{array}{l}\text { Formación } \\
\text { Universitaria }\end{array}$ & $\begin{array}{l}\text { 32. Percepción de estudiantes de } \\
\text { Psicología sobre el uso de facebook }\end{array}$ & $\begin{array}{l}\text { Depto. de Psicología, } \\
\text { Universidad de la }\end{array}$ & Empírico \\
\hline
\end{tabular}

Plataformas digitales en la educación a distancia en México, una alternativa de estudio en comunicación. Arturo S. Hernández, Ennio H. Carro y Isaías Martínez. Página 16 de 27 
RED. Revista de Educación a Distancia. Núm. 59, Artíc. 07, 30-10-2019

DOI: http://dx.doi.org/10.6018/red/60/07

\begin{tabular}{|c|c|c|c|c|c|}
\hline \multirow{6}{*}{ México } & $(\mathrm{CIT}), \mathrm{La}$ & \multirow{6}{*}{$\begin{array}{l}\text { Revista de } \\
\text { Investigación } \\
\text { Educativa } 22\end{array}$} & \multirow{6}{*}{$\begin{array}{l}\text { para desarrollar pensamiento crítico } \\
\text { (Castro y González-Palta, 2016). } \\
\text { 33. La comunidad virtual práctica. } \\
\text { Alternativa para la formación } \\
\text { continua de profesores (Gómez y } \\
\text { Silas, 2016). }\end{array}$} & \multirow{2}{*}{\multicolumn{2}{|c|}{$\begin{array}{l}\text { Serena, Coquimbo, } \\
\text { Chile }\end{array}$}} \\
\hline & $\begin{array}{l}\text { Serena } \\
\text { Instituto de }\end{array}$ & & & & \\
\hline & Investigacione & & & de Estudios Superiores & 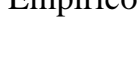 \\
\hline & & & & de Occidente, México, & \\
\hline & $\begin{array}{l}\text { Educación, } \\
\text { Universidad }\end{array}$ & & & & \\
\hline & Veracruzana & & & & \\
\hline \multirow{4}{*}{$\begin{array}{l}\text { Costa } \\
\text { Rica }\end{array}$} & Instituto de & Revista & \multirow{4}{*}{$\begin{array}{l}\text { 34. La orientación del aprendizaje } \\
\text { para la toma estratégica de apuntes } \\
\text { en estudiantes universitarios } \\
\text { (Cabrera, Eguino y Jiménez, 2015). }\end{array}$} & \multirow{10}{*}{$\begin{array}{l}\text { Fac. de Psicología de la } \\
\text { Universidad Central } \\
\text { "Marta Abreu" y } \\
\text { Centro de Superación } \\
\text { para la Cultura, Villa } \\
\text { Clara, Cuba } \\
\text { (Universidad } \\
\text { Pedagógica y } \\
\text { Tecnológica de } \\
\text { Colombia; Universidad } \\
\text { de Boyacá, Colombia) }\end{array}$} & \multirow[t]{5}{*}{ Empírico } \\
\hline & Investigación & Actualidades & & & \\
\hline & en Educación, & Investigativas & & & \\
\hline & $\begin{array}{l}\text { Universidad } \\
\text { de Costa Rica }\end{array}$ & en Educación & & & \\
\hline \multirow{6}{*}{ Colombia } & & & & & \\
\hline & Revista del & \multirow[t]{5}{*}{ Zona Próxima } & \multirow{5}{*}{$\begin{array}{l}\text { 35. Tres dimensiones para la } \\
\text { evaluación de sistemas de gestión } \\
\text { de aprendizaje (LMS) (Ardila y } \\
\text { Ruiz, 2015). }\end{array}$} & & \multirow[t]{5}{*}{ Empírico } \\
\hline & Estudios en & & & & \\
\hline & Educación & & & & \\
\hline & Universidad & & & & \\
\hline & $\begin{array}{l}\text { del Norte, } \\
\text { Barranquilla }\end{array}$ & & & & \\
\hline \multirow[t]{6}{*}{ Chile } & Centro de & \multirow{6}{*}{$\begin{array}{l}\text { Formación } \\
\text { Universitaria }\end{array}$} & \multirow{6}{*}{$\begin{array}{l}\text { 36. Propuesta de modelo para el } \\
\text { proceso de enseñanza-aprendizaje } \\
\text { colaborativo de la observación en } \\
\text { diseño, utilizando la pizarra digital } \\
\text { interactiva (PDI) (Briede, Leal, } \\
\text { Mora y Pleguezuelos, 2015). }\end{array}$} & Depto. de Arte y & \multirow[t]{6}{*}{ Empírico } \\
\hline & Información & & & Tecnologías del & \\
\hline & Tecnológica & & & Diseño- Desarrollo & \\
\hline & (CIT), La & & & Tecnológico y & \\
\hline & Serena & & & pedagógica, & \\
\hline & & & & $\begin{array}{l}\text { Universidad del Bío- } \\
\text { Bío, Chillán, Chile }\end{array}$ & \\
\hline \multirow[t]{5}{*}{ Colombia } & Centro de & Formación & \multirow{5}{*}{$\begin{array}{l}\text { 37. Perfil de aprendizaje y } \\
\text { rendimiento académico en una } \\
\text { asignatura de Química en } \\
\text { modalidad a distancia y presencial } \\
\text { en dos Programas de Ingeniería } \\
\text { (Acevedo, Tirado y Montero, 2015). }\end{array}$} & Fac. de Ingeniería, & \multirow[t]{5}{*}{ Empírico } \\
\hline & Información & Universitaria & & Universidad de & \\
\hline & Tecnológica & & & Cartagena, Colombia & \\
\hline & (CIT), La & & & & \\
\hline & Serena & & & & \\
\hline
\end{tabular}

Fuente: Autoría propia

En la tabla 11, se observa únicamente los cinco artículos empíricos detectados en la tabla 10 y que por cumplir con el criterio de contar con características metodológicas, se describen de manera sintética; no se toman en cuenta los dos artículos teóricos que también se mencionan en la tabla diez, por no cumplir con este criterio. El análisis de los cinco artículos referente a la producción de estudios sobre las plataformas digitales en la educación en México, centra en un $80 \%$ sus investigaciones en la educación superior (universidades o instituciones) y en un $20 \%$ sus estudios en la educación media superior (secundaria), así como en los docentes (80\%) y en estudiantes (20\%); las universidades que más investigación realizan en sus instalaciones es la Universidad de Guadalajara (60\%), la UNAM (20\%) y la Universidad Autónoma del Estado de Hidalgo (UAEH) (20\%); éstas tres universidades mexicanas no aparecen en las diez primeras instituciones que se reporta en la tabla 6, porque, como se ha señalado por razones de espacio, están dentro de un largo listado donde solo se reportan las diez primeras, y de la cual la UAEH se encuentra en el lugar número 14 de la lista, la UNAM ocupa el número 24 de la lista y, la Universidad de Guadalajara, está en el lugar 44 del listado. Por otra parte, las plataformas de uso en el momento que se realizó la

Plataformas digitales en la educación a distancia en México, una alternativa de estudio en comunicación. Arturo S. Hernández, Ennio H. Carro y Isaías Martínez. Página 17 de 27 
RED. Revista de Educación a Distancia. Núm. 59, Artíc. 07, 30-10-2019

DOI: http://dx.doi.org/10.6018/red/60/07

investigación es la Moodle (40\%), la Blackboard (20\%), la Coursera (20\%) y una no reportada $(20 \%)$.

Tabla 11

Cinco artículos empíricos sobre las plataformas digitales en la educación a distancia en México.

\begin{tabular}{|c|c|c|c|c|}
\hline Artículo & Qué investiga & Institución & Grado & Plataforma \\
\hline $\begin{array}{l}\text { 1. Perspectiva docente sobre el } \\
\text { ambiente de aprendizaje en el sistema } \\
\text { de educación a distancia de la } \\
\text { Facultad de Ingeniería en Sistemas de } \\
\text { Producción Agropecuaria (Velázquez } \\
\text { y Martínez, 2011). }\end{array}$ & $\begin{array}{l}\text { Factores y espacios } \\
\text { que influyen en el } \\
\text { ambiente } \\
\text { aprendizaje (aula } \\
\text { centro de cómputo }\end{array}$ & $\begin{array}{l}\text { Facultad de } \\
\text { Ingeniería } \\
\text { en Sistemas } \\
\text { Producción } \\
\text { Agropecua } \\
\text { ria }\end{array}$ & $\begin{array}{l}\text { Educ. } \\
\text { Sup. }\end{array}$ & No reporta \\
\hline $\begin{array}{l}\text { 2. La comunicación y la colaboración } \\
\text { vistas a través de la experiencia en un } \\
\text { MOOC (Vázquez, Bras, Bucio y } \\
\text { Rodríguez, 2017). }\end{array}$ & $\begin{array}{ll}\text { Diseño instruccional } \\
\text { de curso abierto en } \\
\text { línea (MOOCS), en } \\
\text { espacios } & \text { de } \\
\text { colaboración } & \end{array}$ & UNAM & $\begin{array}{l}\text { Educ. } \\
\text { Sup. }\end{array}$ & Coursera \\
\hline $\begin{array}{l}\text { 3. Percepción del profesor sobre el } \\
\text { uso del b-learning para fortalecer } \\
\text { competencias laborales (Ocampo, } \\
\text { Gómez y Zambrano, 2015). }\end{array}$ & $\begin{array}{lll}\text { A un grupo } & \text { de } \\
\text { profesores } & \text { sobre } & \text { el } \\
\text { proceso } & & \text { de } \\
\text { certificación } & \text { en } & \text { b- } \\
\text { learning } & & \end{array}$ & UAEH & $\begin{array}{l}\text { Educ. } \\
\text { Sup. }\end{array}$ & Blackboard \\
\hline $\begin{array}{l}\text { 4. Integración de las tecnologías de la } \\
\text { información y comunicación en un } \\
\text { modelo de enseñanza flexible. El caso } \\
\text { del Centro Universitario de los Valles } \\
\text { de Guadalajara, México (Calderón- } \\
\text { Mayorga, Ruiz-Palmero y Sánchez- } \\
\text { Rodríguez, 2016). }\end{array}$ & $\begin{array}{l}\text { El uso que hacen los } \\
\text { docentes de la } \\
\text { tecnología del Centro } \\
\text { Universitario de los } \\
\text { Valles de Guadalajara }\end{array}$ & U. de G. & $\begin{array}{l}\text { Educ. } \\
\text { Sup. }\end{array}$ & Moodle \\
\hline $\begin{array}{l}\text { 5. La comunidad virtual práctica. } \\
\text { Alternativa para la formación } \\
\text { continua de profesores (Gómez y } \\
\text { Silas, 2016). }\end{array}$ & $\begin{array}{l}\text { Los productos de una } \\
\text { comunidad virtual de } \\
\text { prácticas de las } \\
\text { matemáticas }\end{array}$ & $\begin{array}{l}1^{\circ} \text { grado de } \\
\text { Secundaria } \\
\text { Escuelas } \\
\text { públicas }\end{array}$ & $\begin{array}{l}\text { Educ. } \\
\text { Media }\end{array}$ & Moodle \\
\hline
\end{tabular}

Fuente: Autoría propia

Un análisis más específico de la metodología reporta lo siguiente: El 100\% de los artículos contiene un apartado de método, metodología o contexto y metodología; el $60 \%$ reporta en este apartado el objetivo de la investigación, el $40 \%$ no lo reporta; un 60\% reporta el tipo de enfoque contra un $40 \%$ que no lo reporta (40\% reporta enfoque cualitativo y $20 \%$ mixto). En cuanto al diseño de investigación o de estudio cualitativo, se reporta lo siguiente: un $20 \%$ reporta ser un estudio fenomenológico y otro $20 \%$ reporta ser un diseño secuencial explicativo-diseño secuencial descriptivo (es el que referencia ser un estudio mixto), el 60\% no reporta este apartado; con respecto al tipo de investigación, el 100\% no reporta este apartado; en lo referente a la población o universo, el $100 \%$ si lo reporta: la población de un $20 \%$ son 24 profesores, la de otro $20 \%$ son reporta 22 mil estudiantes inscritos y 13 mil estudiantes activos en línea; otro $20 \%$ reporta 2,800 profesores, otro $20 \%$ reporta a 102 profesores y el $20 \%$ restante reporta a 30 profesores. En cuanto a la muestra y muestreo, un

Plataformas digitales en la educación a distancia en México, una alternativa de estudio en comunicación. Arturo S. Hernández, Ennio H. Carro y Isaías Martínez.

Página 18 de 27 
$20 \%$ reporta como muestra a 30 profesores-actores y que es un muestreo por conveniencia, otro $20 \%$ reporta que no hay muestra pues se investigó el universo total y, el $60 \%$ restante no reporta este apartado; en cuanto al tiempo que duro la investigación el 100\% si reporta este apartado con los siguientes datos: $20 \%$ de febrero a julio de 2012, $20 \%$ reporta solamente semanas sin especificar mes o año, 20\% reporta curso de 2013, 20\% reporta los años 2012 a 2013 y, 20\% reporta de agosto de 2012 a junio de 2013.

Respecto a los estadísticos empleados en la metodología, solamente el $40 \%$ reporta este apartado: $20 \%$ reporta que de la entrevista se hizo la validación y confiabilidad por expertos y que de un cuestionario se hizo una prueba piloto; otro $20 \%$ reporta un análisis univariado y bivariado y que del cuestionario se obtuvo la confiabilidad de 0.857 del Alfa de Cronbach, el $60 \%$ de los artículos no reporta este apartado; por otra parte los programas detectados en los artículos indican que para el análisis de los datos un 20\% utilizó el Excel, un 20\% el SPSS v.20 así como el Atlas Ti y, un 60\% no reporta este apartado.

En cuanto al instrumento de medición, el 100\% lo reporta de la siguiente manera: $20 \%$ aplicó un cuestionario; $20 \%$ detecto a través de la observación a un informante privilegiado, aplicó entrevistas a profundidad y cuestionarios a través del Google; $20 \%$ aplicó cuestionarios en línea a través de la plataforma Limesurvey y entrevistas estructuradas con siete preguntas abiertas; $20 \%$ aplicó entrevistas a profundidad y cuestionarios y; $20 \%$ aplicó bitácoras para los profesores, de las cuales se realizaron análisis de las páginas. Finalmente, del apartado del procedimiento, el 100\% no reportó este apartado.

\section{Discusión}

La investigación de la enseñanza universitaria con apoyo de plataformas digitales en México se enfoca en los espacios y ambientes de aprendizaje virtual, en los espacios de colaboración, en los procesos de certificación de plataformas virtuales para maestros, en el uso que hacen los docentes de la tecnología y, los productos que se generan al usar una plataforma virtual, las cuales están vinculadas y fundamentadas en el campo de la educación y a la tecnología digital como herramienta que apoya al proceso educativo. Por lo que la cuantificación de información general y específica, así como de carácter metodológico de las revisiones sistemáticas son importantes porque el presente estudio pretende generar vínculos con la educación y la tecnología a través de trabajos en el campo de la comunicación educativa que permitan visualizar el proceso de enseñanza-aprendizaje a través del análisis de los mensajes que se dan en las plataformas digitales, si se asimilan o no; cuáles son los contenidos que se proponen; si se elaboraron con el lenguaje adecuado o, si se utilizan los canales que tienen las plataformas virtuales para la transmisión de la información; si las imágenes contienen o no los códigos que requiere el receptor para asimilar los mensajes y sobre todo, qué parte del proceso de la comunicación es susceptible de mejorar. La comparación con otros estudios de comunicación, permite visualizar de manera general que revisiones sistemáticas se han realizado en el campo de la comunicación; desde qué perspectiva se han abordado los estudios, cómo se ha sistematizado la información obtenida, para después conocer el estado general la temática analizada $\mathrm{y}$, de manera específica, si existen estudios sobre los procesos de la comunicación en la educación con apoyo de plataformas digitales.

Plataformas digitales en la educación a distancia en México, una alternativa de estudio en comunicación. Arturo S. Hernández, Ennio H. Carro y Isaías Martínez.

Página 19 de 27 
En este sentido, la presente investigación, se parece a la realizada por Fuentes (1996), quien sistematizó 1,019 documentos de la ciencia de la comunicación, contenidos en la Sistematización Documental de 1986 a 1994 provenientes de la investigación de la Comunicación en México, en donde solo 228 de los 1,019 documentos sistematizados refieren métodos que consideró en esos años como investigaciones empíricas y, de los cuales, el 27.2\% las clasificó como análisis de contenidos (62 documentos); el 20.2\% como encuestas (46 documentos); el $16.2 \%$ las encuadró como etnografía u observación participante (37 documentos); el 11.8\% los consideró un análisis semiótico o del discurso; el 9.6\% las clasificó como sistematización documental (22 documentos) y, el $15.0 \%$ la consideró en otros (solamente 34 documentos).

Contrario a lo realizado por Fuentes, en el presente trabajo se sistematizaron estudios de un tema específico y no general en donde se encontraron 497 artículos que hacían referencia sobre las plataformas digitales en la educación a distancia y siete de ellos en México, lo cual es mucho menor a lo reportado por Fuentes (1996), lo cierto es que, se encontró un mayor número de elementos o características metodológicos en cinco de los siete artículos detectados, que permitieron definir con mayor precisión a los artículos que eran de corte empírico o teórico, de acuerdo al subtítulo empleado para su consideración. Por otra parte, algunos resultados comparables con este estudio sobre la investigación en comunicación de Castillo-Esparcia, Rubio-Moraga y Almansa-Martínez (2012), en el cual se realizó un análisis bibliométrico de las revistas de mayor impacto del ISI y en donde se determina que el idioma inglés (97.7\%) es el más utilizado en las revistas analizadas del ISIWeb como el Journal of Communication, Journal of Health Communication, Public Opinion Quartely, Journal of Computer-Mediate Communication, Human Communication Research, Communication Resarch, Communication Theory y, Interaction Studies y, contrasta con los resultados obtenidos en el presente estudio en donde el idioma más empleado para publicar es el español (62\%), seguido del portugués (21\%) y al último el inglés (17\%), lo anterior debido que las bases digitales analizadas fueron DOAJ, SciELO y Redalyc, así como a la temática investigada relacionada con el país de México y, en los resultados de ambos estudios, es en donde hay similitud en cuanto a la media de autores, siendo de 1.5 autores por artículo en Communication Theory y 3.94 en Journal of Health Communication y, en la presente investigación la media de autores es de 2.52, coincidiendo en que es frecuente observar que un solo artículo es elaborado por una o dos personas, pero también hasta por cuatro o cinco investigadores.

Una limitante fue no hacer una exploración en revistas de educación, de las cuales existe una gran cantidad de ellas en México, Latinoamérica, el Caribe y España, la cual pudo haber brindado mayor información desde el campo de la educación, analizando estudios con tendencias y características hacia la comunicación o los procesos comunicacionales vinculados a la educación en los distintos contextos de enseñanza-aprendizaje. Otra limitante del estudio fueron la muestra, los criterios de búsqueda empleados y las bases de datos empleadas para la búsqueda. La población de 497 artículos encontrados no representaba los estudios sobre las plataformas digitales en la educación a distancia en México, por lo que se recurrió a agrupar en categorías a todos los artículos encontrados y obtener así, una categoría vinculada específicamente a la temática de investigación, cuyo resultado fueron7 artículos

Plataformas digitales en la educación a distancia en México, una alternativa de estudio en comunicación. Arturo S. Hernández, Ennio H. Carro y Isaías Martínez.

Página 20 de 27 
que se tomaron como muestra. Algunos criterios establecidos para la búsqueda de los artículos proporcionaban mucha información con títulos muy ambiguos y otros criterios no arrojaban información. La bases de datos de libre acceso seleccionados para el estudio como DOAJ, SciELO y Redalyc proporcionan una vasta cantidad de artículos, pero solamente son tres bases de datos y se podrían seleccionar más, aunque las publicaciones encontradas en las bases exploradas pueden ser representativas inclusive de aquellas mexicanas que captan el trabajo científico de mayor impacto internacional, ya que las revistas que se encuentran en el Journal Citation Report (JCR) de las misma manera se encuentran en las bases analizadas, al menos en nuestro caso; además de que al revisar las bases de datos DOAJ, SciELO y Redalyc se ampliaron las posibilidades de encontrar artículos en diversas revistas que no se encuentran en el JCR. Una de las fortalezas del estudio fue el análisis de congruencia inter-decodificador para agrupar los artículos vinculados al tema investigado en categorías, por lo que la aplicación del coeficiente de Kappa como procedimiento para determinar la concordancia entre dos codificadores y sus registros de información, le dio mayor consistencia al estudio. La investigación proporcionará la oportunidad de visualizar el contexto sobre lo que se ha investigado sobre las plataformas digitales en la educación a distancia en México, proporcionando con ello, información relevante para los investigadores en la ciencia de la comunicación y el campo de la comunicación educativa y las plataformas de educación a distancia.

\section{Conclusiones}

El objetivo del estudio fue conocer la cantidad de producción de artículos empíricos publicados en el campo de las plataformas digitales en la educación a distancia en México, en los resultados obtenidos se observa que la proporción de artículos empíricos (5) es mayor a la de los artículos teóricos (2), cumpliéndose el objetivo de la investigación y comprobándose la hipótesis nula que plantea que la cantidad de artículos teóricos en el campo de las plataformas digitales en la educación a distancia en México, no es mayor a la de los artículos empíricos. Se concluye que, el número reducido de artículos publicados sobre las plataformas digitales en la educación a distancia en México, dadas las bases de datos consideradas son escasos, sobre todo si agregamos que en México no hay estudios sobre esta temática específicamente en escuelas secundarias a excepción de uno; es contrastante la cantidad de autores, co-autores o colaboradores con las pocas publicaciones sobre la temática abordada indican un mínimo interés de los edu-comunicadores por esta línea de investigación; hay sin embargo, investigadores y universidades en México abocados al estudio de las tecnologías digitales en ambientes educativos. Se proporciona información sobre aspectos educativos referentes a plataformas digitales y comunicativos desde lo que son las revisiones sistemáticas siguiendo métodos objetivos y sistematizados de evaluación, los datos e información resultante serán de importancia ante la falta de revisiones sistemáticas en el campo de la comunicación educativa vinculada a las plataformas digitales. Los resultados servirán como referente para la producción científica sobre la comunicación educativa y las plataformas digitales, señalando áreas de oportunidad en aspectos de índole metodológica, revisiones meta-analíticas y evaluaciones de calidad.

Plataformas digitales en la educación a distancia en México, una alternativa de estudio en comunicación. Arturo S. Hernández, Ennio H. Carro y Isaías Martínez.

Página 21 de 27 
Presentación del artículo: 7 de abril de 2019

Fecha de aprobación: 20 de octubre de 2019

Fecha de publicación: 30 de octubre de 2019

Hernández, A.S., Carro, E.H. y Martínez, I. (2019). Plataformas digitales en la educación a distancia en México, una alternativa de estudio en comunicación. RED. Revista de Educación a Distancia, 60. DOI: http://dx.doi.org/10.6018/red/60/07

\section{Financiación}

Esta investigación no ha recibido ninguna subvención específica de los organismos de financiación en los sectores públicos, comerciales o sin fines de lucro.

\section{Referencias}

Acevedo, D., Tirado, D. y Montero, P. (2015). Perfil de aprendizaje y rendimiento académico en una asignatura de Química en modalidad a distancia y presencial en dos programas de Ingeniería. Formación Universitaria, 8(6), 3-46. doi: 10.4067/S071850062015000600006

Aguilera, E. R. (2014). Carta al Editor. Revista Social Especializada del Dolor, 21(6), 359360. Recuperado de http://scielo.isciii.es/pdf/dolor/v21n6/10_carta.pdf

Andrade, A. E. y Velázquez, G. E. (2014). La Biblioteca de la Facultad de Ingeniería en la plataforma educativa de la UdelaR-Uruguay: implementando un servicio de formación de usuarios virtual. Revista Interamericana de Bibliotecología, 37(2), 171178. Recuperado de http://www.scielo.org.co/pdf/rib/v37n2/v37n2a5.pdf

Ardila-Rodríguez, M. (2011). Indicadores de calidad de las plataformas educativas digitales. Educ.Educ., $\quad$ 14(1), 189-206. Recuperado de http://educacionyeducadores.unisabana.edu.co/sabana/index.php/eye/article/viewArt icle/1836/2414

Ardila, M. J. y Ruiz, C. E. (2015). Tres dimensiones para la evaluación de sistemas de gestión de aprendizaje (LMS). Zona Próxima. Revista del Instituto de Estudios en Educación Universidad del Norte, 22, 69-86. Recuperado de https://www.redalyc.org/pdf/853/85339658006.pdf

Area, M. M. (1991). La tecnología educativa en la actualidad. La evidencia de una crisis. Qurriculum. Revista de Teoría, Investigación y Práctica Educativa, (3), 1-11. Recuperado de http://www.quadernsdigitals.net/index.php?accionMenu=hemeroteca.VisualizaArtic uloIU.visualiza\&articulo_id=2650

Beltrán, O. A. (2005). Revisiones sistemáticas de la literatura. Revista Colombiana de Gastroenterología, 20(1), 60-69. Recuperado de http://www.scielo.org.co/pdf/rcg/v20n1/v20n1a09.pdf

Plataformas digitales en la educación a distancia en México, una alternativa de estudio en comunicación. Arturo S. Hernández, Ennio H. Carro y Isaías Martínez.

Página 22 de 27 
Borgobello, A. y Roselli, N. D. (2016). Rendimiento académico e interacción sociocognitiva de estudiantes en un entorno virtual. Educ. Pesqui, 42(2), 359-374. http://dx.doi.org/10.1590/S1517-9702201606143478

Briede, J., Leal, I., Mora, M. y Pleguezuelos, C. (2015). Propuesta de modelo para el proceso de enseñanza-aprendizaje colaborativo de la observación en diseño, utilizando la pizarra digital interactiva (PDI). Formación Universitaria, 8(3), 15-26. doi: 10.4067/S0718-50062015000300003

Cabrera, R. I., Eguino, de la P. Y. y Jiménez, G. M. (2015). La orientación del aprendizaje para la toma estratégica de apuntes en estudiantes universitarios. Revista Actualidades Investigativas en Educación, 15(1), 1-28. doi: 10.15517/aie.v15i1.16975

Calderón-Mayorga, C., Ruiz-Palmero, J. y Sánchez-Rodríguez, J. (2016). Integración de las tecnologías de la información y comunicación en un modelo de enseñanza flexible. El caso del Centro Universitario de los Valles de Guadalajara, México. Formación Universitaria, 9(5), 37-48. doi: 10.4067/S0718-50062016000500005

Cardona-Arias, J. A., Higuita-Gutiérrez, L. F. y Ríos-Osorio, L. A. (2016). Revisiones sistemáticas de la literatura científica: la investigación teórica como principio para el desarrollo de la ciencia básica y aplicada. doi: 10.16925/9789587600377

Carrillo, A. A. y Mota, M. S. (2010). La asesoría en la enseñanza de las matemáticas en la distancia, utilizando la plataforma Web CT. Revista Didasc@lia. Didáctica y Educación, 3, 63-72. Recuperado de file://I educación a C:/Users/Arturo/Downloads/DialnetLaAsesoriaEnLaEnsenanzaDeLasMatematicasE nLaEducaci-4227294.pdf

Carmona, S. E. y Rodríguez, S. E. (2017). Buenas prácticas en la educación superior virtual a partir de especificaciones de estándares e-Learning. Sophia, 13(1), 13-26. doi: 10.18634/sophiaj.13v.1i345

Castillo-Esparcia, A., Rubio-Moraga, Á., y Almansa-Martínez, A. (2012). La investigación en Comunicación. Análisis bibliométrico de las revistas de mayor impacto del ISI. Revista Latina de Comunicación Social, (67), 248-270. Recuperado de http://www.redalyc.org/articulo.oa?id=81923566006

Castro, P. J. y González-Palta, I. 2016). Percepción de estudiantes de Psicología sobre el uso de facebook para desarrollar pensamiento crítico. Formación Universitaria, 9(1), 4556. doi: 10.4067/S0718-50062016000100006

Cerda, J. y Villarroel, L. (2008). Evaluación de la concordancia inter-observador en investigación pediátrica: Coeficiente de Kappa. Revista Chilena de Pediatría, 79(1), 54-58. Recuperado de

http://www.scielo.cl/scielo.php?script=sci_arttext\&pid=S0370-41062008000100008

Chalela, N. S., Valencia, A. A., Bermúdez, H. J. y Ortega, R. C. (2016). Percepciones estudiantiles acerca del uso de nuevas tecnologías en instituciones de Educación Superior en Medellín. Revista Lassallista de Investigación, 13(2), 151-162. Doi: 10.22507/rli.v13.n2a14

Plataformas digitales en la educación a distancia en México, una alternativa de estudio en comunicación. Arturo S. Hernández, Ennio H. Carro y Isaías Martínez.

Página 23 de 27 
Chiva, S. I., Ramos, S. G., Gómez, D. M. y Alonso, A. A. (2013). La $e$-Evaluación del aprendizaje a través de la plataforma Aula Virtual de la Universitat de València. @atic.Revista d'innovacion educativa, 11, 60-68. doi: 10.7203/attic.11.3051

CONACYT (2019). Manual del Sistema de Clasificación de Revistas Mexicanas de Ciencia $y$ Tecnología (CRMCYT). México: SCIMAGO. Recuperado de http://www.revistascytconacyt.mx/manual-sistema-crmcyt2019.pdf

Córdova, S. M. (2011).Optimización y evaluación de la plataforma virtual continental para mejorar la gestión de tutoría virtual. Apuntes de Ciencia y Sociedad, 01(02), 160-166. Recuperado de http://journals.continental.edu.pe/index.php/apuntes/article/view/37/36

Díaz, V. (2007). Tipos de encuestas considerando la dimensión temporal. Papers: Revista Sociológica, 86, 131-145. Recuperado de http://www.raco.cat/index.php/Papers/article/download/81389/105877

Frías, M., Arce, C. y Flores-Morales, P. (2016).Uso de la plataforma socrative.com para alumnos de Química General. Educación Química.info, 27, 59-66. doi: 10.1016/j.eq.2015.09.003

García, G. J. (2015). Medición de la concordancia. D.F., México: Departamento de Salud Pública. Facultad de Medicina, UNAM. Recuperado de http://www.paginas.facmed.unam.mx/deptos/sp/wpcontent/uploads/2015/10/U5/_an exo8_presconcor_epidin.pdf

García-Peñalvo, F. J. (2017). Revisión sistemática de la literatura en los trabajos de final de Máster y en las Tesis Doctorales. Recuperado de https://www.um.es/documents/378246/2964900/Normas+APA+Sexta+Edici\%C3\% B3n.pdf/27f8511d-95b6-4096-8d3e-f8492f61c6dc

Gómez, L. L. y Silas, C. J. (2016). La comunidad virtual práctica. Alternativa para la formación continua de profesores. Revista de Investigación Educativa, (22), 28-51. Recuperado de https://www.redalyc.org/pdf/2831/283143550003.pdf

Gough, D., Oliver, S. y Thomas, J. (2012). Introducing systematic reviews. En D. Gough, S. Oliver y J. Thomas (Comp.), An introduction to Systematic Reviews (pp. 1-16). Sage.

Imbernón, M. F., Silva, G. P. y Guzmán, V. C. (2011). Competencias en los procesos de enseñanza-aprendizaje virtual y semipresencial. Comunicar, 18(36), 107-114. doi: 10.3916/C36-2011-03-01

Inzunza, 0., Neyem, A., Sanz, M. E., Valdivia, I., Villarroel, M., Farfán, E., Matte, A. y López-Juri, P. (2017). Anatomicis Network: una Plataforma de software educativa basada en la nube para mejorar la enseñanza de la anatomía en la educación médica. Int. J. Morphol, 35(3), 1168-1177. Recuperado de https://scielo.conicyt.cl/pdf/ijmorphol/v35n3/art56.pdf

Letelier, L. M., Manríquez, J. J., y Rada, G. (2005). Revisiones sistemáticas y metaanálisis: ¿son la mejor evidencia? Revista médica de Chile, 133 (2), 246-249. Recuperado de https://scielo.conicyt.cl/pdf/rmc/v133n2/art15.pdf

Plataformas digitales en la educación a distancia en México, una alternativa de estudio en comunicación. Arturo S. Hernández, Ennio H. Carro y Isaías Martínez.

Página 24 de 27 
Lopes, de M. D. y Rizzo, C. C. (2016). Moodle platform for the construction of knowledge in intensive care: an experimental study. Acta Paulista de Enfermagem, 29(4), 381389. doi: 10.1590/1982-0194201600053

López-Morales, M., Celma-Vicente, M., Cano-Caballero, G. M., Quero-Rufián, A. y Rodríguez-López, M. A. (2011). Docencia universitaria semipresencial. Experiencia en el uso de la plataforma virtual SWAD. Educación Médica, 14(4), 229-234. Recuperado de http://scielo.isciii.es/pdf/edu/v14n4/original3.pdf

Mansilla, D. S., Muscia, G. C. y Ugliarolo, E. A. (2013). Campus Virtual y Facebook en el ámbito universitario. ¿Enemigos o aliados en los procesos de enseñanza y aprendizaje? Educación Química, 24(2), 255-259. Recuperado de http://www.revistas.unam.mx/index.php/req/issue/view/2945/showToc

Melella, C. E. (2016). Migración y TIC: Identidades andinas en Facebook. La Trama de la Comunicación, 20(1), 073-088. Recuperado de http://www.redalyc.org/pdf/3239/323944778004.pdf

Meza, P. J. (2013). ¿Cómo brindar servicios de diseminación selectiva de la información (D.S.I.) a través de las plataformas de aprendizaje Virtuales (P.A.V.)?: una propuesta de convergencia de sistemas. E-Ciencias de la Información, 3(2), 1-10. Recuperado de http://revistaebci.ucr.ac.cr/

MIAR. (2019). Matriz de Información para el Análisis de Revistas. Versión 2019 live. Barcelona, España.: Facultat de Biblioteconomía i Documentació. Recuperado de http://miar.ub.edu/

Mombaque, dos S. W., Regina, S. S. y Alves, de A. P. (2018). Carta al Editor. Revista Latino-Americana de Enfermagem, 26, 1-3. doi: 101590/1518-8345.2885.3074

Mora-Vicarioli, F., Hooper-Simpson, C. y Durán-Gutiérrez, Y. (2017). Definición de aspectos procedimentales y buenas prácticas para el servicio de asesoría del programa aprendizaje en línea de la Universidad Estatal a Distancia de Costa Rica. Revista Electrónica Educare, 21(3), 1-28. doi: 10.15359/ree.21311

Moreno, B., Muñoz, M., Cuéllar, J., Domancic, S. y Villanueva, J. (2018). Revisiones sistemáticas: definición y nociones básicas. Revista Clínica Periodoncia Implantología y Rehabilitación Oral, 11(3), 184-186. doi: 10.4067/S071901072018000300184

Núñez, I., Míguez, M. y Seoane, G. (2016). Wikis en Moodle: la mirada de estudiantes y docentes. Educación Química, 27, 257-263. doi: 10.1016/j.eq.2016.08.001

Ocampo, L.A., Gómez, Z. M. y Zambrano, I. D. (2015). Percepción del profesor sobre el uso del b-learning para fortalecer competencias laborales. Apertura, 7(2), 1-12. Recuperado de http://www.scielo.org.mx/pdf/apertura/v7n2/2007-1094-apertura-702-00011.pdf

Pablos De, J. M., Colás, M. P., López, G. A. y García-Lázaro, I. (2019). Los usos de las plataformas digitales en la enseñanza universitaria. Perspectivas desde la investigación educativa. Revista de Docencia Universitaria, 17(1), 59-72. doi: 10.4995/redu.2019.11177

Plataformas digitales en la educación a distancia en México, una alternativa de estudio en comunicación. Arturo S. Hernández, Ennio H. Carro y Isaías Martínez.

Página 25 de 27 
Prado, C., Rodríguez, V. D. y de Almeida, D. M. (2011). Teoría da aprendizagem Signifcativa: elaboração e avaliação de aula virtual na plataforma moodle. Revista Brasileira de Enfermagem, 64(6), 1114-1121. Recuperado de http://www.scielo.br/pdf/reben/v64n6/v64n6a19.pdf

Pérez, C. M. y Saker, B. A. (2012). Análisis de la efectividad del uso de la plataforma virtual webct en el proceso de enseñanza y aprendizaje en la Universidad del Magdalena, Colombia. REXE Revista de Estudios y Experiencias en Educación, 11(21), 89-105. Recuperado de http://www.redalyc.org/articulo.oa?id=243124125006

Quinceno, C. B., Arango, V. S. y Vázquez, L. C. (2016). Profesores frente a estudiantes: las dos orillas de la educación bimodal. Anagramas, 14(28), 15-23. Recuperado de http://www.scielo.org.co/pdf/angr/v14n28/v14n28a01.pdf

Ramírez, V. W. y Barajas, V. J. I. (2017). Uso de las plataformas educativas y su impacto en la práctica pedagógica en instituciones de educación superior de San Luis Potosí. Revista Electrónica de Tecnología Educativa, (60), 1-13. Recuperado de https://edutec.es/revista/index.php/edutec-e/article/view/798/pdf

Repiso, R., Torres, D., Delgado, E. (2011). Análisis bibliométrico y de redes sociales en tesis doctorales españolas sobre televisión (1976/2007). Revista Comunicar, 19(37), 151159. Recuperado de http://www.redalyc.org/articulo.oa?id=15820024018

Rodríguez, D. O. y Pineda, P. R. (2011). Plataforma virtual de péndulo sobre carro deslizante para la enseñanza del control automático. Ingenio Magno, 3, 40-47. Recuperado de http://revistas.ustatunja.edu.co/index.php/ingeniomagno/article/view/62/52

Romero, A. L., Salinas, U. V. y Mortera, G. F. (2010). Estilos de aprendizaje basados en el modelo de Kolb en la educación virtual. Apertura, 2(1), 1-26. Recuperado de https://www.redalyc.org/articulo.oa?id=68820841007

Rubio-Aparicio, M., Sánchez-Meca, J., Marín-Martínez, F. y López-López, J. A. (2018). Recomendaciones para el reporte de revisiones sistemáticas y meta-análisis. Anales de Psicología, 34(2), 412-420. doi: 10.6018/analesps.34.2.320131

Ruíz, R. A. y Gómez, D. D. (2004). Diseño y desarrollo de una plataforma digital de apoyo a la docencia y a la investigación en Archivos Electrónicos: un portal temático. IR Information Research, 9(4). Recuperado de http://www.informationr.net/ir/94/paper194.html

Salvador-Oliván, J. A., Marco-Cuenca, G. y Arquero-Avilés, R. (2018). Las revisiones sistemáticas en biblioteconomía y documentación: análisis y evaluación del proceso de búsqueda. Revista Española de Documentación Científica, 41(2), 1-19. doi: 10.3989/redc.2018.2.1491

Santa, C. C., Calderón, S. C. y Rojas, M. C. (2011). Impacto del uso de la plataforma virtual moodle en el proceso de enseñanza aprendizaje en la Universidad Continental. Apuntes de Ciencia y Sociedad, 01(02), 120-126. Recuperado de http://journals.continental.edu.pe/index.php/apuntes/article/view/32/31

Siegel, S. y Castellan, J. (1995). Estadística no paramétrica aplicada a las ciencias de la conducta. México: Trillas.

Plataformas digitales en la educación a distancia en México, una alternativa de estudio en comunicación. Arturo S. Hernández, Ennio H. Carro y Isaías Martínez.

Página 26 de 27 
Torres, D., Cabezas Á., y Jiménez E. (2013). Altmetrics: nuevos indicadores para la comunicación científica en la Web 2.0. Comunicar, 21(41), 53-60. Recuperado de http://www.redalyc.org/articulo.oa?id=15828675007

Torres, C. P. C. y Cobo, B. J. K. (2017). Tecnología educativa y su papel en el logro de los fines de la educación. Revista Venezolana de Educación (EDUCERE), 21(68), 3140. Recuperado de http://www.redalyc.org/articulo.oa?id=35652744004

Torres-Fonseca, A. y López-Hernández, D. (2014). Criterios para publicar artículos de revisión sistemática. Revista de Especialidades Médico-Quirúrgicas, 19(3), 293-299. Recuperado de http://www.redalyc.org/pdf/473/47332498021.pdf

Torres, S. M. I. (2010). La enseñanza tradicional de las ciencias versus las nuevas tendencias educativas. Revista Electrónica Educare, 14(1), 131-142. Recuperado de https://www.redalyc.org/pdf/1941/194114419012.pdf

Vázquez, L. E., Bras, R. I., Bucio, G. J. y Rodríguez, V. M. (2017). La comunicación y la colaboración vistas a través de la experiencia en un MOOC. Apertura, 9(1), 126-143. doi: 10.18381/Ap.v9n1.942

Valenzuela-Zambrano, B. y Pérez Villalobos, M. V. (2013). Aprendizaje autorregulado a través de la plataforma virtual Moodle. Educ.Educ., 16(1), 66-79. Recuperado de http://educacionyeducadores.unisabana.edu.co/index.php/eye/article/view/2000/303 9

Velázquez, S. G. y Martínez, M. M. (2011). Perspectiva docente sobre el ambiente de aprendizaje en el sistema de educación a distancia de la Facultad de Ingeniería en Sistemas de Producción Agropecuaria. Apertura, 3(1). Recuperado de http://www.udgvirtual.udg.mx/apertura/index.php/apertura/article/view/195/210

Vidal, L. M., Oramas, D. J. y Borroto, C. R. (2015). Revisiones sistemáticas. Revista Cubana de Educación Médica Superior, 29(1), 198-207. Recuperado de http://www.medigraphic.com/pdfs/educacion/cem-2015/cem151s.pdf

Vidrio, T. P., Gómez, Z. M. G. y Zambrano, I. D. (2015). Valoración didáctica del uso de Moodle en la educación media superior. Apertura, 7(1), 1-20. Recuperado de http://www.udgvirtual.udg.mx/apertura/index.php/apertura/article/view/667/426

Zulma, S. M., Vega, F. Y., Nivardy, M. C. y Hernández, E. J. (2017). Diseño e implementación de una plataforma digital para la gestión de tutorías y su impacto en la deserción de estudiantes de nivel superior. Computación e Informática, 1. Recuperado

de https://www.redalyc.org/jatsRepo/5122/512253717008/html/index.html

Plataformas digitales en la educación a distancia en México, una alternativa de estudio en comunicación. Arturo S. Hernández, Ennio H. Carro y Isaías Martínez.

Página 27 de 27 